\title{
Fungal and bacterial nematicides in integrated nematode management strategies
}

\author{
Mahfouz M. M. Abd-Elgawad ${ }^{1 *}$ and Tarique Hassan Askary ${ }^{2}$
}

\begin{abstract}
Plant-parasitic nematodes (PPNs) pose a serious threat to quantitative and qualitative production of many economic crops worldwide. An average worldwide crop loss of 12.6\% (equaled $\$ 215.77$ billion) annually has been estimated due to these nematodes for only the top 20 life-sustaining crops. Due to the growing dissatisfaction with hazards of chemical nematicides, interest in microbial control of PPNs is increasing and biological nematicides are becoming an important component of environmentally friendly management systems. Fungal and bacterial nematicides rank high among other biocontrol agents. In order to maximize their benefits, such bio-nematicides can be included in integrated nematode management (INM) programs, and ways that make them complimentary or superior to chemical nematode management methods were highlighted. This is especially important where bio-nematicides can act synergistically or additively with other agricultural inputs in integrated pest management programs. Consolidated use of bio-nematicides and other pesticides should be practiced on a wider basis. This is especially important, since there are many bio-nematicides which are or are likely to become widely available soon. Identification of research priorities for harnessing fungal and bacterial nematicides in sustainable agriculture as well as understanding of their ecology, biology, mode of action, and interaction with other agricultural inputs is still needed. Therefore, accessible fungal and bacterial nematicides with their comprehensive references and relevant information, i.e., the active ingredient, product name, type of formulation, producer, targeted nematode species and crop, and country of origin, are summarized herein.
\end{abstract}

Keywords: Nematodes, Biocontrol, Bio-nematicides, Integrated pest management, Synergism

\section{Background}

Plant-parasitic nematodes (PPNs) are considered hidden enemy of the farmers as the nematodes are subterranean in habitats and growers are unaware of losses caused by them. Much of the damage caused by nematodes goes unreported or is often confused with other causes such as fungal attack, water stress, or other physiological disorders, and by the time the disease is diagnosed, the loss to crops has already been incurred by these tiny organisms. A great loss to crops has been reported in quantitative, qualitative, and monetary terms. Abd-Elgawad and Askary (2015) reported an average worldwide crop loss of $12.6 \%$ (equaled $\$ 215.77$ billion), due to these nematodes for only the top 20

\footnotetext{
* Correspondence: mahfouzian2000@yahoo.com

'Plant Pathology Department, National Research Centre, El-Behoos St., Dokki, Giza 12622, Egypt

Full list of author information is available at the end of the article
}

life-sustaining crops based on the 2010-2013 production figures and prices. Moreover, 14.45\% (\$142.47 billion) was an average annual yield loss in the subsequent group of food or export crops. These figures are astonishing, and the authentic figure, when more crops throughout the world are considered, probably exceeds such estimations. At the same time, numerous relevant and challenging issues have been demonstrating the desperate need of human beings to provide more and better food for an over-populated world. Abd-Elgawad (2014) stressed the importance of such issues due to deregistration and banning of effective nematicides available because of environmental and health hazards, renewable manifestation of resistance-breaking nematode pathotypes on many important crops, climate change, increased adoption of intensive agriculture, and potential occurrence of quarantine nematodes. Therefore, nematode management and research should be continuously 


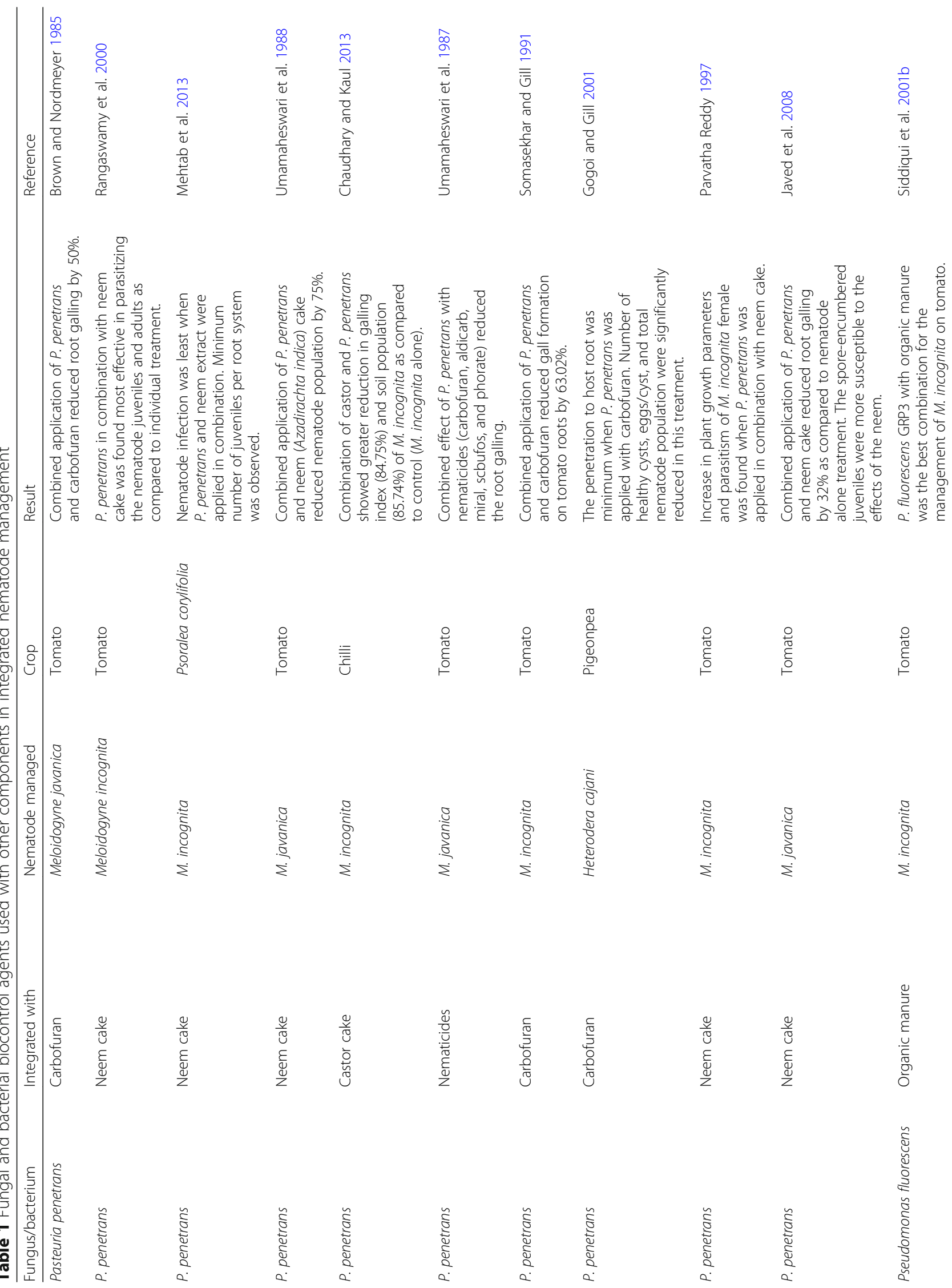




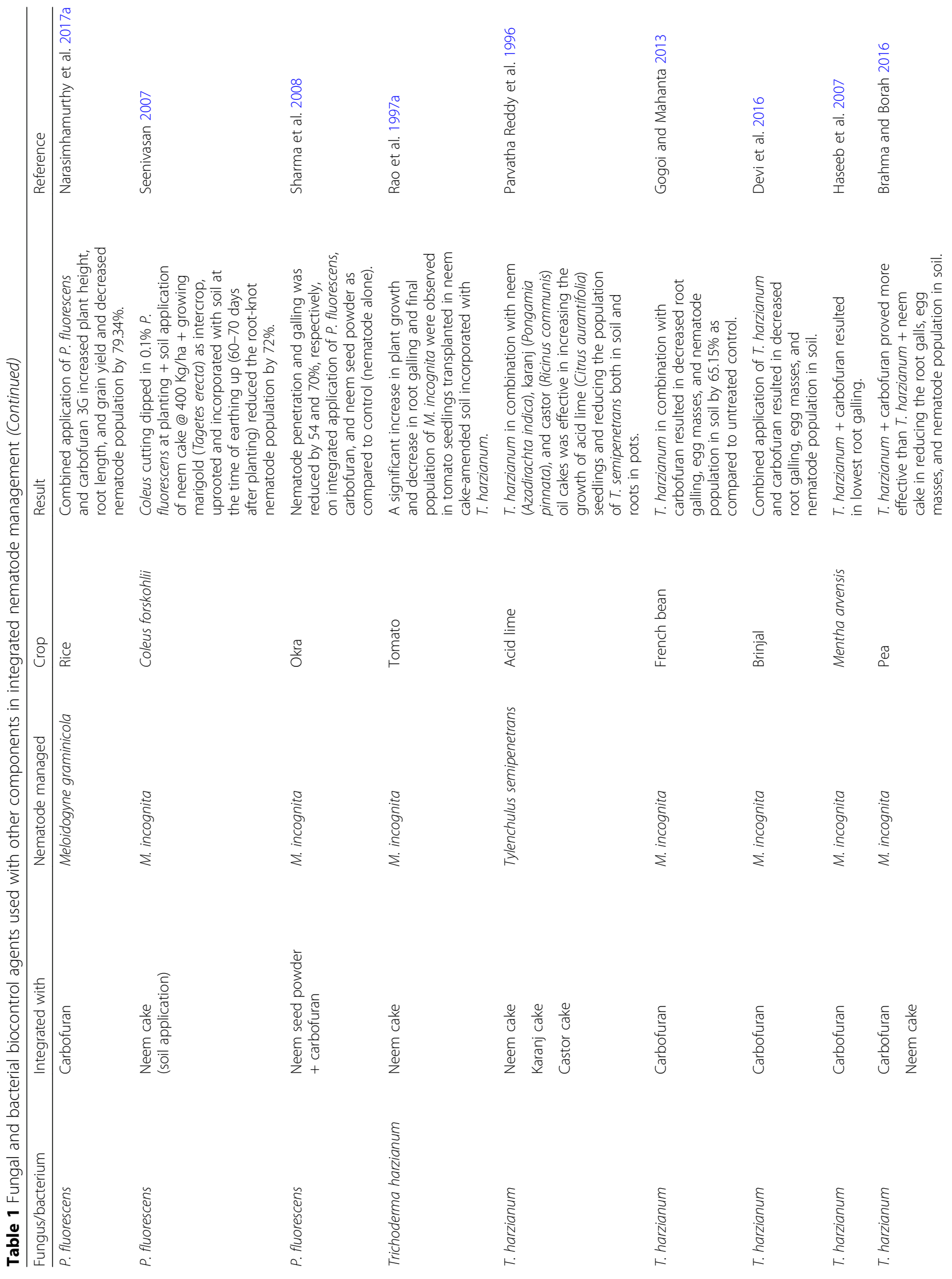




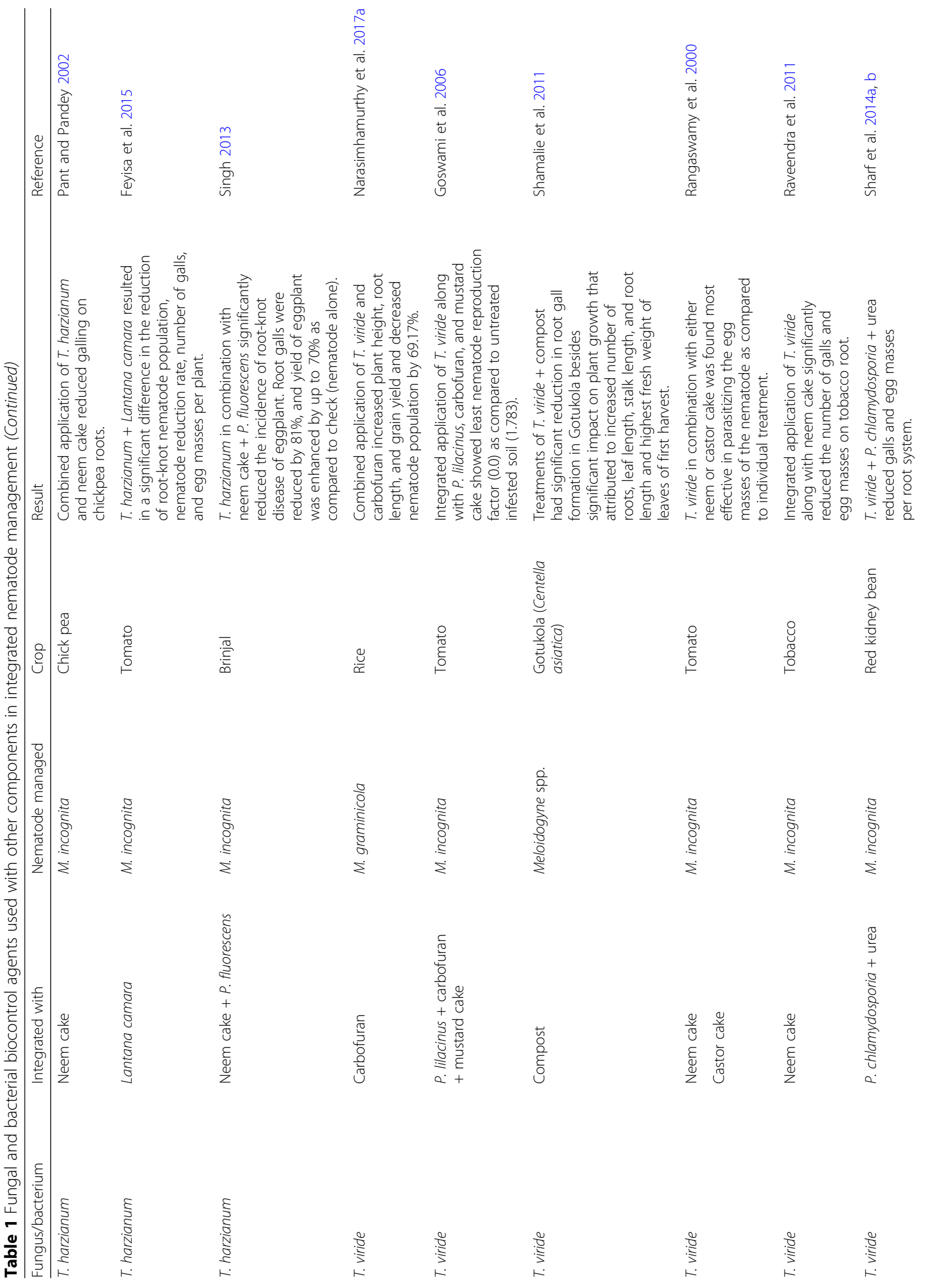




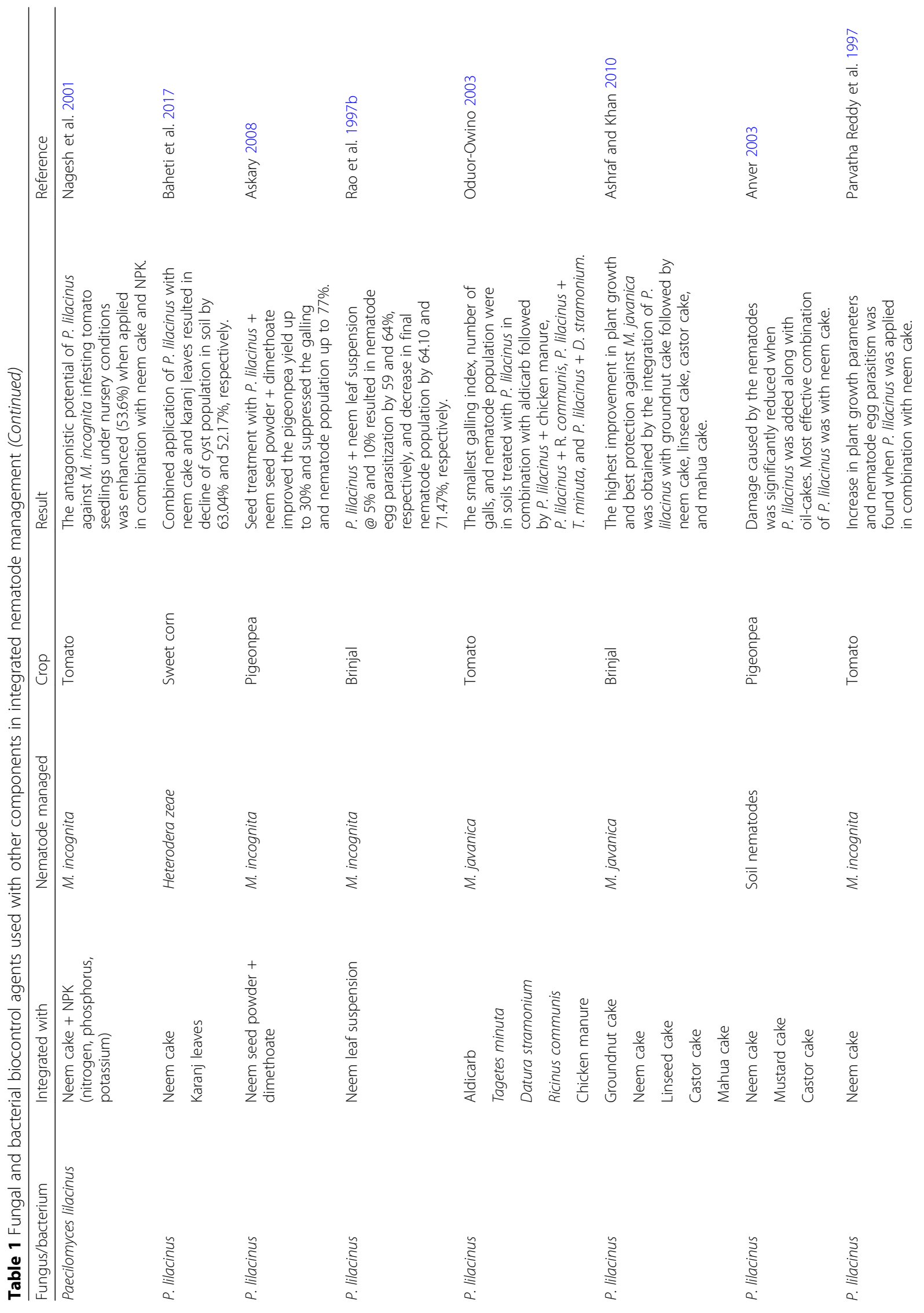




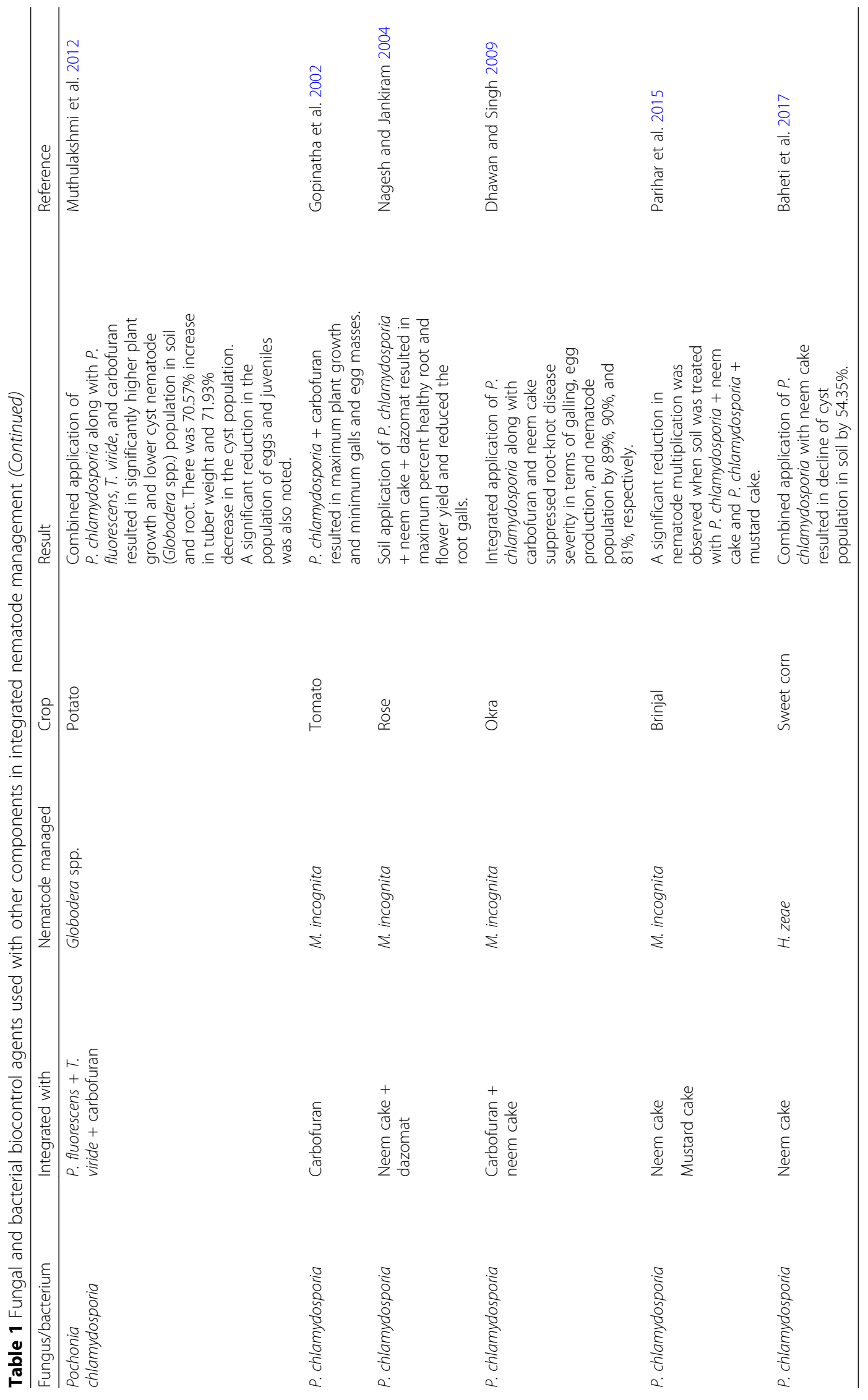


refined and oriented to offer better control of PPNs in an environmentally and economically beneficial manner.

Importance of biological control of pests is growing, and this is obviously reflected by considerable venture capital in research by multinational firms and also by their acquisitions of small biotechnology corporations with microbial product portfolios (Wilson and Jackson 2013). Bio-products containing antagonists of fungi and bacteria rank high among other bio-nematicides (Askary 2015a, b; Eissa and Abd-Elgawad 2015). As such nematicides represent living systems, a number of difficulties exist to develop commercial bio-nematicidal products. Problems with their culture and formulation, variable gap between laboratory and field performance, potential negative effects on non-target or beneficial organisms, and expectations of broad-spectrum activity and quick efficacy based on practice with synthetic chemical nematicides have been addressed in details by some workers (Glare et al. 2012; Askary and Martinelli 2015). Rapid progress has been made during the past two decades in different aspects of bio-nematicidal production and use. This was especially important for the development of in vitro mass culture concerning Pasteuria spp. and innovative, easy-to-use formulations of numerous products. Yet, there is still a desperate need to poise these bio-nematicides as more effective and reliable products against PPNs. This trend is currently materialized in a variety of approaches, including studies on their applications, improved shelf-life, mass-culture, and interaction with other biotic and abiotic factors as well as integration of biocontrol with other management techniques.

The objective of this review article is to highlight the current knowledge of the biological control potential of fungal and bacterial agents in attempt to include them in effective integrated nematode management (INM) programs. Also, research priorities and perceived factors for harnessing fungal and bacterial nematicides in sustainable agriculture were identified.

\section{Fungal and bacterial interaction with other inputs}

The most studied and promising groups among the nematode-antagonistic organisms are the nematophagous fungi and bacteria (Askary and Martinelli 2015). The two groups include many species. These bio-nematicides are frequently applied to sites and ecosystems that routinely receive other inputs including chemical pesticides, surfactants (e.g. wetting agents), fertilizers, mineral nutrition, and soil amendments which may interact with bio-active ingredients targeting PPNs. Fungal and bacterial biocontrol agents used with other components in INM are listed in Table 1. Basically, such biocontrol agents (BCA) are living systems sensitive to biotic and abiotic factors that result from inputs, especially in the soil rhizosphere. Thus, BCA should be compatible with them to maximize their benefits. In this respect, we propose that bio-nematicides should not be used as direct competitors with chemical nematicides for several reasons. Bio-nematicides fall behind chemical nematicides in traits prized by growers: price, performance, handling, distribution, and ease of both storage and use. Hence, our suggestion is especially important where bio-nematicides can act synergistically or additively with such inputs in INM programs. Positive results have been demonstrated (Table 1). For example, shoot dry weight of tomato had better $(P \leq 0.05)$ increase, when Pseudomonas fluorescens GRP3 was combined with organic manure for the management of Meloidogyne

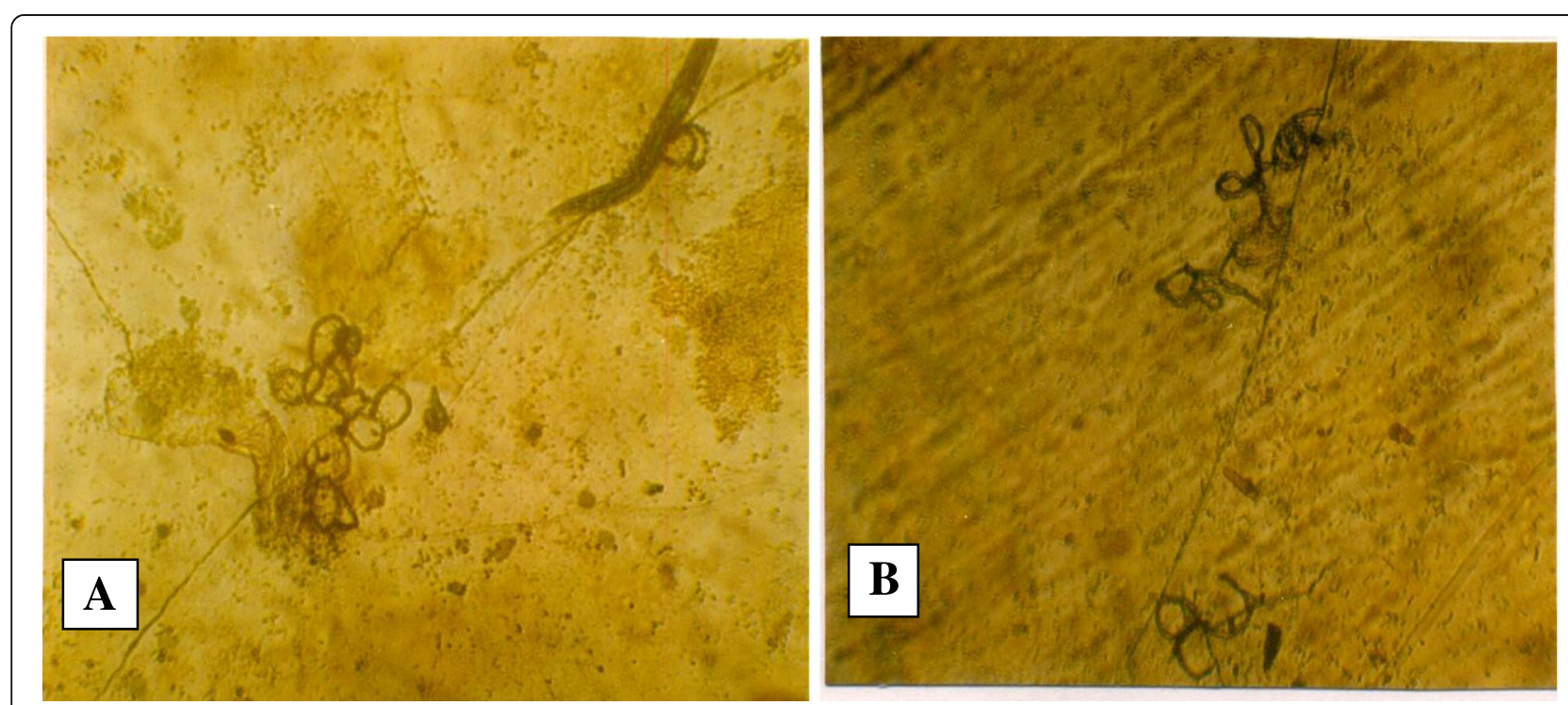

Fig. 1 Monacrosporium megalosporum. a A portion of hypha showing entangled nematode with adhesive net, the lower portion showing arched or circular hyphal meshes. b A portion of hypha with adhesive network 


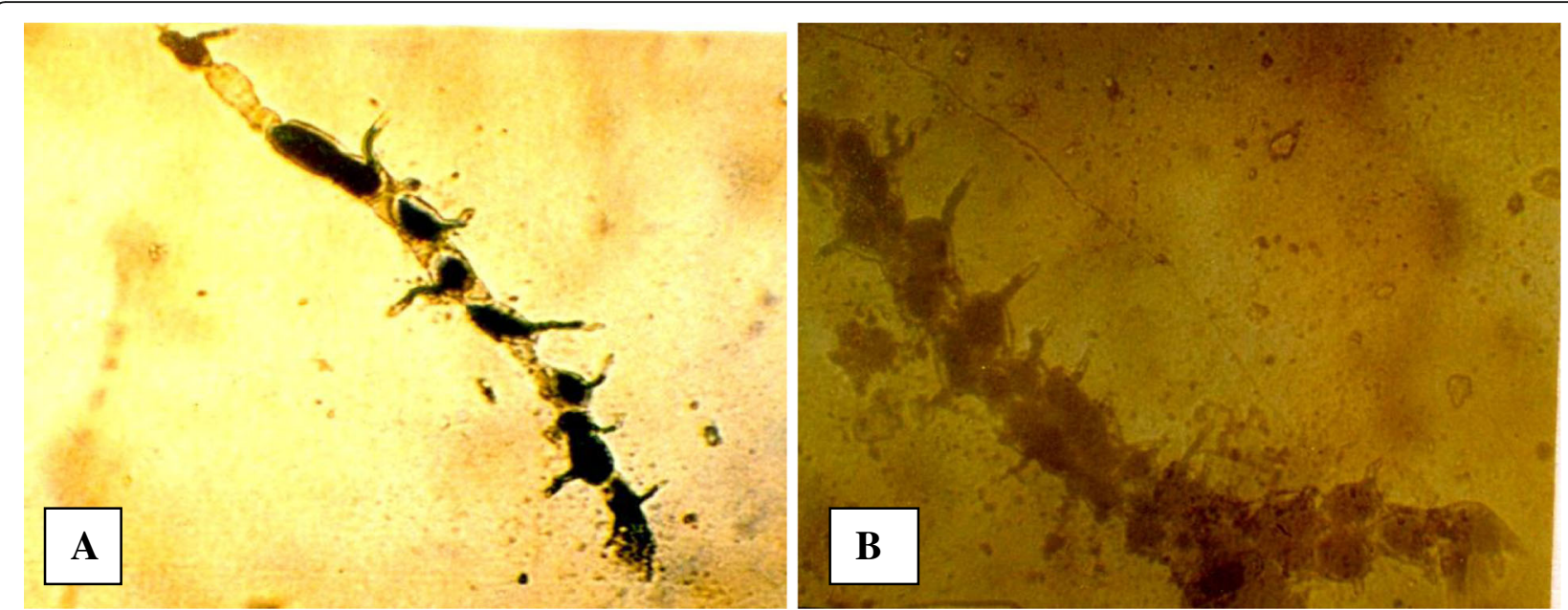

Fig. 2 Catenaria anguillulae. a Chain of mature and immature sporangium. b Double chain of mature and immature sporangium on nematode cadaver

incognita than using either P. fluorescens or organic manure alone (Siddiqui et al. 2001b). New tactics for synergistically or even additively incorporating BCA with favorable inputs should be tried further and broadly disseminated for real and better penetration of markets and developed BCA. A similar plan was recently put forward for insecticides too (Stevens and Lewis 2017).

We should also highlight and get use of approaches where bio-nematicides can be included in INM programs in ways that make them complimentary or superior to chemical pest management methods. In this respect, endospores formed by bacterial genera Bacillus, Clostridium, and Pasteuria are tolerant to exposures for most agrochemicals. Such endospore-forming bacteria are both the most heat-resistant form of life and highly resistant to desiccation and chemical destruction; these endospores have a prolonged shelf life (more than a year) and can also be applied to seeds several days before planting. They can be used along with inorganic and organic fertilizers, microelements, and several fungicides, herbicides, and pesticides; they can often be tank-mixed. Abd-Elgawad and Vagelas (2015) focused on widening this approach since a tank mix of one or more inputs with a bio-nematicide can save time and money. Also, bio-nematicides can also be used in rotation with such chemicals as pesticides to delay pest resistance by breaking pressure from a single mode of action. Clearly, consolidated use of bio-nematicides and other pesticides should be practiced on a wider basis. In this vein, only few companies are actively fostering the concerted use of bio-nematicides and chemical pesticide, e.g., the product $\mathrm{VOTiVO}^{\mathrm{mm}}$ and $\mathrm{PONCHO}^{\circ} / \mathrm{VOTiVO}^{\mathrm{m}}$ mix, which is based on Bacillus firmus against PPNs, combined with a synthetic insecticide, Poncho1, as a seed treatment (Anonymous 2018).

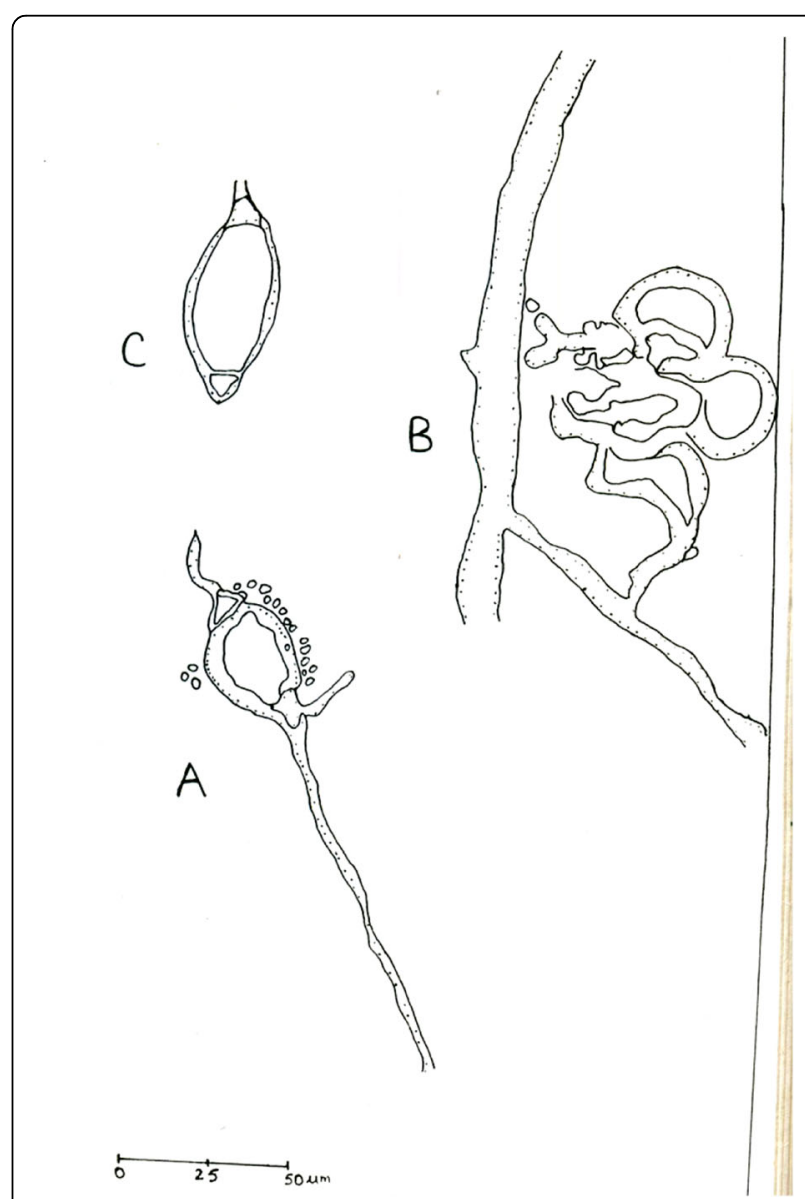

Fig. 3 Catenaria anguillulae. A. A conidium on agar surface under germination. B. A portion of hypha with adhesive network. C. Detached conidium 


\section{Mode of action of fungal and bacterial nematicides}

Fungi group may be divided into nematode-trapping, endoparasitic, egg- and female-parasitic, and toxin-producing fungi (Askary 1996; Jansson et al. 1997). For example, for the nematode-trapping fungus, entangled nematode with adhesive network of Monacrosporium megalosporum hypha is illustrated (Fig. 1). Catenaria anguillulae, an endoparasitic fungus, is a member of the Chytridiomycota, the only major group of true (chitin-walled) fungi that produce motile spores, termed zoospores (Deacon 2018). This fungus is often found as a facultative (non- specialized) parasite of nematodes and other small organisms. Phase-contrast microscopy was used to show the single and double chain of mature and immature fungal sporangium on parasitized nematodes (Fig. 2). It can be grown easily on culture media and different parts, under germination, of the fungus grown on agar surface (Fig. 3). Based on their modes of action, the nematophagous bacteria can also be broadly grouped into parasitic bacteria and non-parasitic rhizobacteria. Eissa and Abd-Elgawad (2015) adopted the following categories of nematophagous bacteria: obligate parasitic bacteria,

Table 2 Modes of action of fungal and bacterial biocontrol agents against phytonematodes (Askary and Martinelli 2015)

Mode of action

Fungus

Aspergillus niger

Paecilomyces lilacinus

Trichoderma harzianum

T. viride

Pochonia chlamydosporia

Bacteria

Pasteuria penetrans

Pseudomonas fluorescens

Bacillus firmus

B. thuringiensis

B. subtilis
It is an egg parasite and induces systemic resistance against plant-parasitic nematodes. The fungus coming in contact with a cyst or an egg mass begins to grow rapidly. It colonizes the eggs where larval formation has not been completed, thus providing early protection to the growing plants against nematodes.

It is mainly egg parasite. The fungus produces antibiotics viz., leucinostatin and lilacin and enzymes such as protease and chitinase. Protease has nematicidal activity, causes degradation of the eggshell, and inhibits hatching. Chitinase breaks down the eggshell making the route for the fungus to pass through. The decomposition of chitin releases ammonia, which is toxic to second-stage juveniles of root-knot nematode (RKN). Its hypha enters the vulva and anus of RKN females. The fungus penetrates the egg and develops profusely inside and over the eggs, completely inhibiting juvenile development. The infected eggs swell and buckle. As penetration continues, the vitelline layer of the egg splits into three bands and a large number of vacuoles; lipid layer disappears at this stage. The developing juvenile inside the egg is destroyed by the rapidly growing hyphae. Many conidiophores are produced and the hypha moves to the adjacent eggs.

Secretes many lytic enzymes like chitinase, glucanases, and proteases which help parasitism of Meloidogyne and Globodera eggs. The chitin layer is dissolved through enzymatic activity. The hyphae of T. harzianum penetrate the eggs and juvenile cuticle, proliferate within the organism, and produce toxic metabolites.

Produce antibiotics like trichodermin, dermadin, trichoviridin, and sesquiterpene heptalic acid which are involved in the suppression of nematodes.

Parasitizes the eggs and adult females of plant-parasitic nematodes. The root-knot and cyst nematodes are the primary hosts of this fungus, but it is also known to parasitize citrus, burrowing, and reniform nematodes. The fungus enters the nematode cysts either through natural openings or it may directly penetrate the wall of the cyst. It forms a branched mycelia network when in close contact with the smooth eggshell. The fungus produces an appressorium that adheres to the eggshell by mucigens and from which an infection peg develops and penetrates the eggshell. Penetration also occurs from lateral branches of the mycelium. This results in disintegration of the eggshell's vitelline layer and also partial dissolution of the chitin and lipid layers, possibly due to the activity of exoenzymes. Egg hatching is inhibited due to toxins secreted by the fungus.
Bacterial spores are attached to the nematode's body and germinate forming a germ tube that penetrates the body cuticle. Vegetative mycelial colonies eventually fill the body with a large number of endospores.

Produce antibiotics viz., phenazines, tropolone, pyrrolnitrin, pyocyanin, and 2,4-diacetylphloroglucinol which have suppressive effect on plant-parasitic nematodes.

Enzymatic action, degradation of root exudates, root-protection, and the production of a phytohormone.

Nematicidal toxins found in families of $B$. thuringiensis proteins.

The genes are encoding surfactin and iturin synthesis as antibiotics. 
opportunistic parasitic bacteria, rhizobacteria, cry protein-forming bacteria, endophytic bacteria, and symbiotic bacteria.

Several nematicides have been banned due to their health and environmental hazards; therefore, the merits and demerits of potential biological control agents with different modes of action against PPNs should be continuously researched for more details about their virulence mechanisms. The modes of action for common fungal and bacterial nematicides are

Table 3 List of commercial products of fungal biocontrol agents used in the nematode management (data are collected by the authors and based on Askary and Martinelli (2015))

\begin{tabular}{|c|c|c|c|c|}
\hline Fungus & Product & Formulation & Company/institution & Country \\
\hline Aspergillus niger & Kalisena & $\begin{array}{l}\text { Soluble (liquid) } \\
\text { concentrate }\end{array}$ & Cadila Pharmaceutical Limited & India \\
\hline A. niger & Kalisena & $\begin{array}{l}\text { Suspension concentrate } \\
\text { for direct application }\end{array}$ & Cadila Pharmaceutical Limited & India \\
\hline A. niger & Beej Bandhu & Wettable powder & - & India \\
\hline A. niger & Pusa Mrida & Wettable powder & - & India \\
\hline A. niger & Kalasipahi & Capsule & - & India \\
\hline Pochonia chlamydosporia & KlamiC ${ }^{\circledast}$ & Granulate & $\begin{array}{l}\text { Rothamsted Research and Centro } \\
\text { Nacional de Sanidad Agropecuaria }\end{array}$ & UK, Cuba \\
\hline P. chlamydosporia & PCMR-1 strain & Liquid & Clamitec, Myco solutions, Lda & Portugal \\
\hline P. chlamydosporia & Xianchongbike & Liquid & $\begin{array}{l}\text { Laboratory for Conservation and } \\
\text { Utilization of Bio-resources, Yunnan } \\
\text { University }\end{array}$ & China \\
\hline P. chlamydosporia & IPP-21 & - & - & Italy \\
\hline Purpureocillium lilacinus & BIOACT ${ }^{\circledR G}$ & $\begin{array}{l}\text { Water-dispersible } \\
\text { granulate }\end{array}$ & Bayer Crop Science & USA \\
\hline P. lilacinus & BIOACT'WP & $\begin{array}{l}\text { Water-dispersible } \\
\text { powder }\end{array}$ & Bayer Crop Science & USA \\
\hline P. lilacinus & PL Gold & Wettable powder & BASF Worldwide, Becker Underwood & South Africa \\
\hline P. lilacinus & Stanes Bio Nematon & Liquid or powder & $\begin{array}{l}\text { Imported from T. Stanes and Company } \\
\text { Limited, India, by Gaara company, Egypt }\end{array}$ & India and Egypt \\
\hline P. lilacinus & PL 251 & $\begin{array}{l}\text { Water-dispersible } \\
\text { granulate }\end{array}$ & Biological Control Products & South Africa \\
\hline P. lilacinus & BIOCON & Wettable powder & Asiatic Technologies Incorporation & Philippines \\
\hline P. lilacinus & Shakti Paecil & Wettable powder & Shakti Biotech & India \\
\hline P. lilacinus & PAECILO ${ }^{\oplus}$ & Wettable powder & Agri Life & India \\
\hline P. lilacinus & Paecilon & Liquid & Enpro Bio Sciences Private Limited & India \\
\hline P. lilacinus & Nematofree & Wettable powder & International Panaacea Limited & India \\
\hline P. lilacinus & Gmax bioguard & Talc based carrier & Greenmax Agro Tech & India \\
\hline P. lilacinus & Yorker & Wettable powder & Agriland Biotech & India \\
\hline P. lilacinus & Miexianning & Talc based carrier & $\begin{array}{l}\text { Agricultural Institute, Yunan Academy of } \\
\text { Tobacco Science }\end{array}$ & China \\
\hline P. lilacinus & $\begin{array}{l}\text { PI Plus }^{\oplus}(P \text {. lilacinus } \\
\text { strain 251) }\end{array}$ & Wettable powder & Biological Control Products & South Africa \\
\hline P. lilacinus & Melocon ${ }^{\oplus}$ WG & $\begin{array}{l}\text { Water-dispersible } \\
\text { granulate }\end{array}$ & Prophyta GmbH Certis & Germany, USA \\
\hline Trichoderma harzianum & Romulus & Wettable powder & DagutatBiolab & South Africa \\
\hline T. harzianum & $\mathrm{ECOSOM}^{\oplus}$ & Wettable powder & Agri Life & India \\
\hline T. harzianum & Trichobiol & Wettable powder & $\begin{array}{l}\text { Control Biologico Integrado; Mora } \\
\text { Jaramillo Arturo Orlando_-Biocontrol }\end{array}$ & Columbia \\
\hline T. harzianum & Commander Fungicide & Wettable powder & H.T.C Impex Private Limited & India \\
\hline T. viride & Trifesol & Wettable powder & Biocultivos Agricultura Sostenible & Columbia \\
\hline
\end{tabular}


Table 4 Biocontrol agents of fungal species targeting nematodes on economic crops

\begin{tabular}{|c|c|c|c|}
\hline Fungus & Nematode managed & Crop & Reference \\
\hline Aspergillus niger & Meloidogyne incognita & Mung bean & Bhat and Wani 2012 \\
\hline A. niger & Meloidogyne spp. & Tomato & Li et al., 2011 \\
\hline A. niger & Meloidogyne javanica & Tomato & Zareen et al. 2001 \\
\hline A. niger & M. javanica & Pigeonpea & Askary 2012 \\
\hline A. niger & Meloidogyne arenaria & Tomato & Mokbel et al. 2009 \\
\hline A. niger & M. incognita & Okra & Sharma et al. 2005 \\
\hline A. niger & Meloidogyne spp. & Tomato & Singh et al. 1991 \\
\hline A. niger & Meloidogyne spp. & Tomato & Khan et al. 1984 \\
\hline A. niger & M. incognita & Brinjal & Goswami and Singh 2002 \\
\hline A. niger & M. javanica & Chickpea & Hussain et al. 2001 \\
\hline Paecilomyces lilacinus & Meloidogyne graminicola & Rice & Narasimhamurthy et al. 2017a, b \\
\hline P. lilacinus & M. incognita & Black gram & Kumar et al. 2017 \\
\hline P. lilacinus & M. incognita & Chrysanthemum & Nagesh et al. 2003 \\
\hline P. lilacinus & M. incognita & Banana & Devrajan and Rajendran 2002 \\
\hline P. lilacinus & M. incognita & Okra & Saikia and Roy 1994 \\
\hline P. lilacinus & M. incognita & Okra & Davide and Zorilla 1986 \\
\hline P. lilacinus & M. incognita & Okra & Simon and Pandey 2010 \\
\hline P. lilacinus & M. incognita & Tobacco & Ramakrishnan and Nagesh 2011 \\
\hline P. lilacinus & M. javanica & Tomato & Ganaie and Khan 2010 \\
\hline P. lilacinus & M. javanica & Tomato & Maheswari and Mani 1989 \\
\hline P. lilacinus & M. incognita & Pittosporum tobira (mock orange) & Baidoo et al. 2017 \\
\hline P. lilacinus & Rotylenchulus reniformis & Tomato & Walters and Barker 1994 \\
\hline P. lilacinus & R. reniformis & Chickpea & Ashraf and Khan 2008 \\
\hline P. lilacinus & M. incognita & Cardamom & Eapen and Venugopal 1995 \\
\hline P. lilacinus & Heterodera cajani & Pigeonpea & Siddiqui and Mahmood 1995 \\
\hline P. lilacinus & Radopholus similis & Banana & Mendoza et al. 2004 \\
\hline \multirow[t]{3}{*}{ P. lilacinus } & M. javanica & Tomato & \multirow[t]{3}{*}{ Khan et al. 2006} \\
\hline & Rotylenchulus similis & Banana & \\
\hline & Heterodera avenae & Barley & \\
\hline P. lilacinus & M. incognita & Tomato & Oclarit and Cumagun 2009 \\
\hline P. lilacinus & M. incognita & Tomato & Khan and Goswami 2000 \\
\hline P. lilacinus & R. similis & Betelvine & Sosamma et al. 1994 \\
\hline P. lilacinus & R. similis & Arecanut & Sudha et al. 2000 \\
\hline P. lilacinus & M. incognita & Tomato & Candanedo-Lay et al. 1982 \\
\hline P. lilacinus & M. incognita & Tomato & Roman and Rodriguez-Marcano 1985 \\
\hline P. lilacinus & Meloidogyne spp. & Lettuce & Prakob et al. 2009 \\
\hline P. lilacinus & M. incognita & Tobacco & Ramakrishnan and Rao 2013 \\
\hline P. lilacinus & M. javanica & Tomato & Khan and Esfahani 1990 \\
\hline P. lilacinus & M. javanica & Pumpkin, Guar, Chili, Watermelon & Perveen et al. 1998 \\
\hline P. lilacinus & Tylenchulus semipenetrans & Citrus Jambhiri & Deka et al. 2002 \\
\hline P. lilacinus & M. incognita & Tomato & Goswami and Mittal 2004 \\
\hline P. lilacinus & M. incognita & Bitter gourd & Bhat et al. 2009 \\
\hline P. lilacinus & M. incognita & Brinjal & Nisha and Sheela 2016 \\
\hline P. lilacinus & M. incognita & Okra, Tomato & Walia et al. 1999 \\
\hline
\end{tabular}


Table 4 Biocontrol agents of fungal species targeting nematodes on economic crops (Continued)

\begin{tabular}{|c|c|c|c|}
\hline Fungus & Nematode managed & Crop & Reference \\
\hline P. lilacinus & T. semipenetrans & Citrus (KhasiMandarin) & Mahanta et al. 2016 \\
\hline P. lilacinus & T. semipenetrans & Citrus (KhasiMandarin) & Manzoor et al. 2002 \\
\hline P. lilacinus & M. incognita & Tomato & Cabanillas and Barker 1989 \\
\hline P. lilacinus & M. incognita & Tomato & Khalil et al. 2012a \\
\hline P. lilacinus & M. incognita & Tomato & Khalil et al. 2012b \\
\hline P. lilacinus & M. incognita & Tomato & Amin 2000 \\
\hline P. lilacinus & M. incognita & Tomato & Cabanillas et al. 1989 \\
\hline P. lilacinus & M. incognita & Okra & Thakur and Devi 2007 \\
\hline P. lilacinus & H. cajani & Pigeonpea & Siddiqui et al. 1998 \\
\hline P. lilacinus & M. javanica & Tobacco & Hewlett et al. 1988 \\
\hline P. lilacinus & M. javanica & Tomato & Esfahani and Pour 2006 \\
\hline \multirow[t]{2}{*}{ P. lilacinus } & M. incognita acrita & Potato & Jatala et al. 1979 \\
\hline & Globodera pallida & & \\
\hline P. lilacinus & M. incognita & Potato & Jatala et al., 1980 \\
\hline P. lilacinus & M. javanica & Pigeonpea & Askary 2012 \\
\hline P. lilacinus & M. incognita & Banana & Jonathan and Rajendran 2000 \\
\hline P. lilacinus & M. incognita & Betelvine & Jonathan et al. 1995 \\
\hline P. lilacinus & M. incognita & Cowpea & Hasan 2004 \\
\hline P. lilacinus & M. incognita & Okra & Dhawan et al. 2004 \\
\hline P. lilacinus & M. incognita & Betelvine & Bhatt et al. 2002a \\
\hline P. lilacinus & R. reniformis & Tomato & Parvatha Reddy and Khan 1988 \\
\hline P. lilacinus & R. reniformis & Chickpea & Anver and Alam 1999 \\
\hline P. lilacinus & R. reniformis & Pigeonpea & Anver and Alam, 1997 \\
\hline P. lilacinus & M. javanica & Broad bean, Okra & Zareen et al. 1999 \\
\hline P. lilacinus & M. arenaria & Brinjal & Sivakumar et al. 1993 \\
\hline P. lilacinus & M. incognita & French bean & Santin 2008 \\
\hline Pochonia chlamydosporia & M. incognita & Tomato & Silva et al. 2017 \\
\hline P. chlamydosporia & M. javanica & Lettuce & Viggiano et al. 2015 \\
\hline P. chlamydosporia & M. javanica & Tomato and Pepper & Tzortzakakis 2007 \\
\hline P. chlamydosporia & M. incognita & Tomato & De Leij et al. 1992 \\
\hline P. chlamydosporia & M. javanica & Broad bean, Okra & Zareen et al. 1999 \\
\hline P. chlamydosporia & Heterodera schachtii & Sugar beet & Ebrahim et al. 2008 \\
\hline P. chlamydosporia & M. incognita & Brinjal & Parihar et al. 2015 \\
\hline P. chlamydosporia & M. incognita & Bell pepper & Rao et al. 2004 \\
\hline P. chlamydosporia & M. incognita & Tomato & Sankaranarayanan et al. 2000 \\
\hline P. chlamydosporia & H. cajani & Pigeonpea & Siddiqui and Mahmood 1995 \\
\hline P. chlamydosporia & Heterodera avenae & Wheat & Bhardwaj and Trivedi 1996 \\
\hline P. chlamydosporia & Meloidogyne hapla & Tomato & De Leij et al. 1993 \\
\hline P. chlamydosporia & M. javanica & Lettuce and Tomato & Verdejo-Lucas et al. 2003 \\
\hline P. chlamydosporia & M. incognita & Okra & Kumar and Jain 2010a \\
\hline P. chlamydosporia & M. incognita & Okra & Dhawan and Singh 2010 \\
\hline P. chlamydosporia & M. javanica & Tomato & Siddiqui and Ehteshamul-Haque 2000 \\
\hline P. chlamydosporia & M. incognita & Pigeonpea & Askary, 2008 \\
\hline P. chlamydosporia & M. incognita & Common bean & Sharf et al. 2014a \\
\hline
\end{tabular}


Table 4 Biocontrol agents of fungal species targeting nematodes on economic crops (Continued)

\begin{tabular}{|c|c|c|c|}
\hline Fungus & Nematode managed & Crop & Reference \\
\hline P. chlamydosporia & M. incognita & Brinjal & Rao et al., 2003 \\
\hline P. chlamydosporia & M. incognita & Brinjal & Dhawan et al. 2008 \\
\hline P. chlamydosporia & R. reniformis & Cotton & Wang et al. 2005 \\
\hline P. chlamydosporia & M. hapla & Lettuce & Viaene and Abawi 2000 \\
\hline P. chlamydosporia & M. incognita & Lettuce and Tomato & Van Damme et al. 2005 \\
\hline P. chlamydosporia & H. cajani & Pigeonpea & Kumar and Prabhu 2008 \\
\hline Trichoderma harzianum & M. javanica & Tomato & Feyisa et al. 2016 \\
\hline T. harzianum & H. cajani & Pigeonpea & Kumar and Prabhu 2008 \\
\hline T. harzianum & M. javanica & Tomato & Naserinasab et al. 2011 \\
\hline T. harzianum & Meloidogyne spp. & Cardamom & Anonymous 1995 \\
\hline T. harzianum & M. incognita & Chickpea & Hemlata et al. 2002 \\
\hline T. harzianum & M. incognita & Chickpea & Pant and Pandey 2002 \\
\hline T. harzianum & M. incognita & Pea & Brahma and Borah 2016 \\
\hline T. harzianum & M. arenaria & Maize & Windham et al. 1989 \\
\hline T. harzianum & M. incognita & Brinjal & Devi et al. 2016 \\
\hline T. harzianum & M. incognita & Cardamom & Eapen and Venugopal 1995 \\
\hline T. harzianum & H. cajani & Pigeonpea & Siddiqui and Mahmood 1996 \\
\hline T. harzianum & M. incognita & French bean & Gogoi and Mahanta 2013 \\
\hline T. harzianum & M. incognita & Brinjal & Kumar and Chand 2015 \\
\hline T. harzianum & M. incognita & Green gram & Deori and Borah 2016 \\
\hline T. harzianum & M. incognita & Pigeonpea & Askary 2008 \\
\hline T. harzianum & M. incognita & Tomato & Kumar and Khanna 2006 \\
\hline T. harzianum & M. javanica & Tomato & Sharon et al. 2001 \\
\hline T. harzianum & M. incognita & Brinjal & Rao et al. 1998 \\
\hline T. harzianum & M. graminicola & Paddy & Pathak and Kumar 2003 \\
\hline T. harzianum & M. incognita & Green gram & Singh and Mahanta 2013 \\
\hline T. harzianum & M. javanica & Tomato & Jamshidnejad et al. 2013 \\
\hline T. harzianum & Meloidogyne spp. & Tomato & Khattak and Khattak 2011 \\
\hline T. harzianum, T. viride & M. incognita & Okra & Kumar and Jain 2010b \\
\hline T. harzianum, T. viride & M. incognita & Okra & Prasad and Anes 2008 \\
\hline T. harzianum, T. viride & M. javanica & Tomato & Al-Hazmi and Javeed 2016 \\
\hline T. harzianum, T. viride & M. incognita & Tomato & Dababat et al. 2006 \\
\hline T. harzianum, T. viride & M. incognita & Tomato & Devi and Sharma 2002 \\
\hline T. harzianum, T. virens & M. graminicola & Rice & Pathak et al. 2005 \\
\hline T. harzianum, T. viride & M. incognita & Tomato & Dababat and Sikora 2007 \\
\hline T. harzianum, T. viride & M. javanica & Mung bean, Okra & Siddiqui et al. 2001a \\
\hline T. harzianum, T. viride & Meloidogyne spp. & Roundleaf fountain Palm & Jegathambigai et al. 2011 \\
\hline \multirow[t]{2}{*}{ T. harzianum, T. viride } & M. javanica & Brinjal & Bokhari 2009 \\
\hline & R. reniformis & & \\
\hline T. viride & M. incognita & Mulberry & Muthulakshmi et al. 2010 \\
\hline T. viride & M. incognita & Tomato & Goswami and Mittal 2004 \\
\hline T. viride & M. incognita & Soybean & Devi and Hassan 2002 \\
\hline T. viride & M. incognita & Chickpea & Pandey et al. 2003 \\
\hline T. viride & Helicotylenchus multicinctus & Banana & Jonathan et al. 2004 \\
\hline
\end{tabular}


Table 4 Biocontrol agents of fungal species targeting nematodes on economic crops (Continued)

\begin{tabular}{|c|c|c|c|}
\hline Fungus & Nematode managed & Crop & Reference \\
\hline T. viride & M. incognita & Okra & Chatali et al. 2003 \\
\hline T. viride & Pratylenchus thornei & Chickpea & Dwivedi et al. 2008 \\
\hline T. viride & M. incognita & Cucumber & Krishnaveni and Subramanian 2004 \\
\hline T. viride & M. incognita & Tomato & Rangaswamy et al. 2000 \\
\hline T. viride & M. incognita & Mulberry & Muthulakshmi and Devrajan 2015 \\
\hline T. viride & M. incognita & Betelvine & Bhatt et al. 2002b \\
\hline T. viride & M. graminicola & Rice & Priya 2015 \\
\hline T. viride & M. incognita & Green gram & Umamaheswari et al. 2004 \\
\hline T. viride & M. incognita & Sugar beet & Kavitha et al. 2007 \\
\hline \multirow[t]{2}{*}{ T. viride } & M. incognita & Cowpea & Kumar et al. 2011 \\
\hline & R. reniformis & & \\
\hline T. virens & M. incognita & Bell pepper & Meyer et al. 2001 \\
\hline Trichoderma spp. & M. incognita & Common bean & Santin 2008 \\
\hline
\end{tabular}

summarized in Table 2. Predatory and egg-parasitic fungi, as well as the parasitic bacteria Pasteuria spp., were the most studied due to their PPN control potential, ease of laboratory production, and adaptation capability under different agricultural systems. Such bioagents, with different action mechanisms, can play a significant role in PPN management. They have a determined specificity against certain species or even stages of PPNs (Askary and Martinelli 2015). Hence, by considering and identifying such a specificity, PPN management can be targeted successfully. Clearly, sometimes, there is a significant difference in the effectiveness of a definite biocontrol agent against the same PPN species. Possible explanations for these differences include loss of virulence during the in vitro culture process or during formulation, or environmental factors occurring in the field (Crow et al. 2011). Also, current investigations of such mechanisms may lack in the exactitude of the applied parameter. Biochemical measures may be more accurate than others. Korayem et al. (1993) examined the effects of the plant extracts of Artemisia absinthium, Citrullus colocynthis, Punica granatum, Ricinus communis, and Thymus vulgaris on motility of Helicotylenchus dihystera and Meloidogyne incognita and the reversibility of the movement inhibition, the egg-hatching inhibition of $M$. incognita, and the inhibition of acetylcholinesterase (ACHEs) of $H$. dihystera. Surprisingly, AChE inhibition by extracts of $P$. granatum, $T$. vulgaris, and A. absinthium were more than that by oxamyl, which was reported as a strong inhibitor for AChE (Opperman and Chang 1990). Likewise, detail information is required regarding the modes of action of many bio-nematicides in terms of their effect on nematode acetylcholinesterase inhibition.

\section{Available products of fungal and bacterial biocontrol agents used against PPNs}

In the past three decades, research workers have prepared different types of formulation of bionematicides that have been commercialized in the world market. Lists of some available fungal (Tables 3 and 4) and bacterial (Tables 5 and 6) nematicides, which indicate relevant information in terms of the active ingredient, product name, type of formulation, producer, targeted nematode species, crop, and country, are presented. There are also cottage industries, which use cheap labor to produce other unavailable microbial products mainly for domestic markets. These products of developing countries, especially in Asia, Africa, and Latin America, might be cost-effective and efficacious against PPNs. However, they have not usually undergone the strict and cost rules of registration schemes required in North America and Europe (Wilson and Jackson 2013). There are also some unpublished products sold on market, but their sell scale is either small, local, and/or has not been approved by the government, while other products are in the pipeline. Therefore, a globally standard procedure for approval by governments, especially for non-registered, available bio-nematicides, was suggested.

Due to their ability to manage a wide range of PPN species, some BCA have been formulated in a commercial product to control different PPN species effectively via a single natural product rather than multiple chemical products (Askary and Martinelli 2015). Coating seeds with biopesticides is an inexpensive option that allows targeted delivery and potentially enhances rhizosphere colonization, but this delivery option requires 
Table $\mathbf{5}$ List of commercial products of bacterial biocontrol agents used in the management of plant-parasitic nematodes

\begin{tabular}{|c|c|c|c|}
\hline Bacterium & Product name & Company/institution & Country \\
\hline \multirow[t]{2}{*}{ Pasteuria penetrans } & \multirow[t]{2}{*}{ Econem } & Nematech & Japan \\
\hline & & Pasteuria Bioscience & USA \\
\hline P. nishizawae & Clariva PN & Syngenta & Brazil \\
\hline P. usage (or P. penetrans) & Econem & Bayer CropScience & Multi-national \\
\hline Pseudomonas fluorescens & SHEATHGUARD (or Sudozone) & Agriland Biotech & India \\
\hline $\begin{array}{l}\text { Bacillus cereus (CM-1c strain) and } \\
\text { Bacillus subtilis (CM-5 strain) }\end{array}$ & BioStart $^{\mathrm{TM}}$ Defensor & Bio-Cat Microbials & USA \\
\hline Bacillus thuringiensis & Avid 0.15EC (or abamectin) & Syngenta & Multinational \\
\hline Bacillus subtilis & Stanes Sting & $\begin{array}{l}\text { Imported from T. Stanes and Company } \\
\text { Limited, India, by Gaara company, Egypt }\end{array}$ & India and Egypt \\
\hline $\begin{array}{l}\text { B. licheniformis } \\
\text { B. subtilis }\end{array}$ & Quartzo & FMC Química do Brasil Ltda. & Brazil \\
\hline $\begin{array}{l}\text { B. licheniformis } \\
\text { B. subtilis }\end{array}$ & Nemix C & FMC Química do Brasil Ltda. & Brazil \\
\hline $\begin{array}{l}\text { B. licheniformis strain FMCHO01(DSM32154) } \\
\text { B. subtilis strain FMCH0O2 (DSM32155) }\end{array}$ & Presense & FMC Química do Brasil Ltda. & Brazil \\
\hline B. firmus & $\begin{array}{l}\text { 1. Bionem-WP } \\
\text { 2. BioSafe-WP } \\
\text { 3. Chancellor }\end{array}$ & Agro Green & Israel \\
\hline B. firmus strain GB-126 & VOTIVO ${ }^{\circledR} \mathrm{WP}$ & Bayer Crop Science & Germany \\
\hline B. methylotrophicus & Onix & $\begin{array}{l}\text { Laboratorio de Bio Controle } \\
\text { Farroupilha S.A. }\end{array}$ & Brazil \\
\hline B. subtilis & Pathway Consortia ${ }^{\circledast}$ & Pathway Holdings & USA \\
\hline $\begin{array}{l}\text { B. chitinosporus, B. laterosporus, } \\
\text { B. licheniformis (mixture) }\end{array}$ & $\begin{array}{l}\text { BioStart }{ }^{\oplus} \\
\text { BioStart }^{\text {MM }}\end{array}$ & $\begin{array}{l}\text { Bio-Cat } \\
\text { Rincon-Vitova }\end{array}$ & USA \\
\hline B. amyloliquefaciens & Nemacontrol & $\begin{array}{l}\text { Simbiose Indústria e Comércio de } \\
\text { Fertilizantes e Insumos }\end{array}$ & Brazil \\
\hline $\begin{array}{l}\text { Bacillus sp., Pseudomonas sp., } \\
\text { Rhizobacterium sp., Rhizobium sp. }\end{array}$ & Micronema & Agricultural Research Centre & Egypt \\
\hline B. cereus & Xian Mie & $\begin{array}{l}\text { XinYi Zhong Kai Agro- Chemical } \\
\text { industry CO., Ltd. }\end{array}$ & China \\
\hline Bacillus spp. & Nemix & Chr. Hansen & Brazil \\
\hline Burkholderia cepacia & Deny Blue circle & Stine Microbial Products & USA \\
\hline $\begin{array}{l}\text { Serratia marcescens produces volatile } \\
\text { metabolites toxic to and other PPNs }\end{array}$ & Nemaless & Agricultural Research Centre & Egypt \\
\hline
\end{tabular}

improved efficacy of coating materials and technology or better formulation. Microbial seed treatment is used for disease control, PPN management, and also for insect control (Glare et al. 2012). Improved seed supply systems that reduce the storage period are required if this delivery mechanism is to become more familiar with BCA. Such multiple effects should be further investigated then materialized commercially.

Different formulations of the same pesticide may generally differ in their toxicity to target organisms. Owing to the continuous introduction of novel active ingredients, carriers, and formulations in different market segments and differences in susceptibility and reaction of bacterial species to nematicide formulations, comprehensive information about different aspects of relevant modules should be available to stakeholders and updated continuously. Current issues in experimentations of biological control agents and their applications against PPNs to maximize their benefits have been recently reviewed (Abd-Elgawad, 2016).

\section{Future prospects}

It should be clear that the use of bio-nematicides is not limited to beneficial BCA, but they should involve the use of their genes and/or products, such as metabolites, that reduce the negative effects of PPNs and promote positive responses by the growing plant. Furthermore, 
Table 6 List of bacterial biocontrol agents against phytonematodes infesting agricultural crops

\begin{tabular}{|c|c|c|c|}
\hline Bacterium & Nematode managed & Crop & Reference \\
\hline \multirow[t]{2}{*}{ Pasteuria penetrans } & Meloidogyne incognita & Tomato, cucumber & Kokalis-Burelle 2015 \\
\hline & Meloidogyne arenaria & Snapdragon & \\
\hline P. penetrans & M. arenaria & Tomato, oriental melon & Cho et al. 2000 \\
\hline P. penetrans & Meloidogyne spp. & Brinjal, mung bean & Zaki and Maqbool 1990 \\
\hline P. penetrans & Heterodera cajani & Cowpea & Singh and Dhawan 1994, 1996, 1999 \\
\hline P. penetrans & Meloidogyne spp. & Sugarcane & Spaull 1984 \\
\hline P. penetrans & M. incognita & Tobacco, soybean, hairy vetch & Brown et al. 1985 \\
\hline P. penetrans & M. incognita & Tomato & Vargas et al. 1992 \\
\hline \multirow[t]{3}{*}{ P. penetrans } & M. incognita & Kiwi & Verdejo-Lucas 1992 \\
\hline & M. arenaria & & \\
\hline & Meloidogyne hapla & & \\
\hline P. penetrans & Xiphinema diversicaudatum & Peach & Ciancio 1995 \\
\hline P. penetrans & M. arenaria & Peanut & Chen et al. 1996, Chen et al. 1997 \\
\hline P. penetrans & M. javanica & Chickpea & Sharma 1992 \\
\hline P. penetrans & M. javanica & Tomato & Maheswari and Mani 1989 \\
\hline \multirow[t]{3}{*}{ P. penetrans } & M. incognita & Tomato & Mankau and Prasad 1972 \\
\hline & M. javanica & & \\
\hline & Pratylenchus scribneri & & \\
\hline \multirow[t]{2}{*}{ P. penetrans } & M. javanica & Tomato & Stirling 1984 \\
\hline & & Grape & \\
\hline P. penetrans & M. arenaria & Peanut, rye, and vetch & Oostendorp et al. 1991 \\
\hline P. penetrans & M. acronea & Tomato & Page and Bridge 1985 \\
\hline P. penetrans & M. incognita & Banana, tomato & Jonathan et al. 2000 \\
\hline P. penetrans & M. incognita & Tomato & Chand and Gill 2002 \\
\hline P. penetrans & M. javanica & Tomato & Daudi et al. 1990 \\
\hline P. penetrans & M. javanica & Tomato & Daudi and Gowen 1992 \\
\hline P. penetrans & M. incognita & Tomato & De Channer 1989 \\
\hline P. penetrans & M. incognita & $\begin{array}{l}\text { Tobacco, soybean, tomato, } \\
\text { hairy vetch, pepper }\end{array}$ & Dube and Smart 1987 \\
\hline P. penetrans & M. graminicola & Tomato & Duponnois et al. 1997 \\
\hline \multirow[t]{2}{*}{ P. penetrans } & M. incognita & Tomato & Weibelzahl-Fulton et al. 1996 \\
\hline & M. javanica & & \\
\hline P. penetrans & M. incognita & Tomato & Adiko and Gowen, 1994 \\
\hline P. penetrans & M. incognita & Brinjal, tomato, wheat & Ahmed 1990 \\
\hline P. penetrans & M. graminicola & Rice & Thakur and Walia 2016 \\
\hline P. penetrans & M. incognita & Tomato & Ahmed et al. 1994 \\
\hline P. penetrans & H. avenae & Wheat & Bhattacharya and Swarup 1988 \\
\hline P. penetrans & M. javanica & Tomato & Walia 1994 \\
\hline P. penetrans & M. incognita & Tomato & De Leij et al. 1992 \\
\hline P. penetrans & Pratylenchus penetrans & Tomato & Somasekhar and Gill 1991 \\
\hline P. penetrans & M. incognita & Tomato & Amin 2000 \\
\hline P. penetrans & M. incognita & Tomato & Rangaswamy et al. 2000 \\
\hline P. penetrans & M. incognita & Tomato & Sekhar and Gill 1991 \\
\hline P. penetrans & M. incognita & Tomato & Ravichandra and Reddy 2008 \\
\hline
\end{tabular}


Table 6 List of bacterial biocontrol agents against phytonematodes infesting agricultural crops (Continued)

\begin{tabular}{|c|c|c|c|}
\hline Bacterium & Nematode managed & Crop & Reference \\
\hline P. penetrans & M. javanica & Tomato & Ciancio and Bourijate 1995 \\
\hline P. penetrans & M. javanica & Grape & Walker and Watchtel 1989 \\
\hline P. penetrans & M. javanica & Tomato & Jayaraj and Mani 1988 \\
\hline P. penetrans & M. incognita & Tomato & Brown and Smart 1985 \\
\hline P. penetrans & M. incognita & Tomato & Singh et al. 2008 \\
\hline P. penetrans & M. incognita & Cherry tomato & Kasumimoto et al. 1993 \\
\hline P. penetrans & M. javanica & Tomato & Walia and Dalal 1994 \\
\hline P. penetrans & M. javanica & Brinjal & Kumar et al. 2005a, b \\
\hline P. penetrans & M. javanica & Okra, chickpea & Vikram and Walia 2015 \\
\hline P. penetrans & M. javanica & Tomato & Vikram and Walia 2014 \\
\hline Pseudomonas fluorescens & M. graminicola & Rice & Narasimhamurthy et al. 2017a \\
\hline P. fluorescens & M. graminicola & Rice & Narasimhamurthy et al. 2017b \\
\hline P. fluorescens & M. incognita & Field pea & Siddiqui et al. 2009 \\
\hline P. fluorescens & M. graminicola & Rice & Seenivasan et al. 2012 \\
\hline P. fluorescens & M. incognita & Tomato & Santhi and Sivakumar 1995 \\
\hline P. fluorescens & H. cajani & Pigeonpea & Siddiqui et al. 1998 \\
\hline P. fluorescens & M. incognita & Okra & Devi and Dutta 2002 \\
\hline P. fluorescens & M. incognita & Bell pepper & Rao et al. 2004 \\
\hline P. fluorescens & M. incognita & Tomato, brinjal & Anita and Rajendran 2002 \\
\hline P. fluorescens & M. incognita & Cowpea & Nama and Sharma 2017 \\
\hline P. fluorescens & M. incognita & Chilli & Wahla et al. 2012 \\
\hline P. fluorescens & M. incognita & Tomato & Siddiqui et al. 2001b \\
\hline \multirow[t]{2}{*}{ P. fluorescens } & Radopholus similis & Banana & Aalten et al. 1998 \\
\hline & Meloidogyne spp. & & \\
\hline P. fluorescens & M. javanica & Tomato & Eltayeb 2017 \\
\hline P. fluorescens & M. incognita & Tomato & Singh and Siddiqui 2010 \\
\hline Pseudomonas sp. & M. incognita & Black pepper & Devapriyanga et al. 2012 \\
\hline P. fluorescens & M. incognita & Mulberry & Muthulakshmi et al. 2010 \\
\hline P. fluorescens & M. incognita & Papaya & Rao 2007 \\
\hline Pseudomonas sp. & Globodera rostochiensis & Potato & Trifonova et al. 2014 \\
\hline P. fluorescens & M. incognita & Maize & Ashoub and Amara 2010 \\
\hline P. fluorescens & Hirschmanniella gracilis & Rice & Seenivasn and Lakshmanan 2002 \\
\hline P. fluorescens & M. incognita & Okra & Kumar and Jain 2010b \\
\hline P. fluorescens & M. arenaria & Groundnut & Kalaiarasan et al. 2010 \\
\hline P. fluorescens & M. incognita & Banana & Sandeep 2004 \\
\hline P. fluorescens & M. javanica & Tomato & Verma 2009 \\
\hline P. fluorescens & Aphelenchoides besseyi & Tuberose & Pathak and Khan 2010 \\
\hline \multirow[t]{2}{*}{ P. fluorescens } & M. incognita & Cowpea & Kumar et al. 2011 \\
\hline & Rotylenchulus reniformis & & \\
\hline P. fluorescens & M. incognita & Banana & Jonathan et al. 2006 \\
\hline P. fluorescens & R. similis & Banana & Kumar et al. 2008 \\
\hline P. fluorescens & R. similis & Banana & Senthilkumar et al. 2008 \\
\hline P. fluorescens & Helicotylenchus multicinctus & Banana & Jonathan et al. 2004 \\
\hline P. fluorescens & M. javanica & Tomato & Siddiqui and Shaukat 2003 \\
\hline
\end{tabular}


Table 6 List of bacterial biocontrol agents against phytonematodes infesting agricultural crops (Continued)

\begin{tabular}{|c|c|c|c|}
\hline Bacterium & Nematode managed & Crop & Reference \\
\hline P. fluorescens & R. reniformis & Cotton & Jayakumar et al. 2004 \\
\hline P. fluorescens & R. reniformis & Cotton & Jayakumar et al. 2002 \\
\hline P. fluorescens & M. incognita & Cotton, cucumber & Hallmann et al. 1998 \\
\hline P. fluorescens & M. incognita & Tomato & Hanna et al. 1999 \\
\hline P. fluorescens & Hirschmanniella gracilis & Rice & Ramakrishnan et al. 1998 \\
\hline P. fluorescens & Tylenchulus semipenetrans & Citrus & Santhi et al. 1999 \\
\hline P. fluorescens & M. incognita & Black pepper & Eapen et al. 1996 \\
\hline P. fluorescens & M. incognita & Grapevine & Shanthi et al. 1998 \\
\hline P. fluorescens & M. incognita & Tomato & Khalil et al. 2012b \\
\hline P. fluorescens & M. incognita & Cucumber & Krishnaveni and Subramanian 2004 \\
\hline P. fluorescens & M. incognita & Sugar beet & Kavitha et al. 2007 \\
\hline P. fluorescens & M. incognita & Okra & Sharma et al. 2008 \\
\hline P. fluorescens & Pratylenchus thornei & Chickpea & Dwivedi et al. 2008 \\
\hline P. fluorescens & M. incognita & Black gram & Akhtar et al. 2012 \\
\hline P. fluorescens & Globodera spp. & Potato & Mani et al. 1998 \\
\hline P. fluorescens & H. cajani & Sesamum indicum & Kumar et al. 2005a, b \\
\hline P. fluorescens & M. incognita & Tomato & Jothi et al. 2003 \\
\hline P. fluorescens & M. graminicola & Rice & Anita and Samiyappan 2012 \\
\hline P. fluorescens & M. graminicola & Rice & Priya 2015 \\
\hline P. fluorescens & M. incognita & Jasmine & Seenivasan and Poornima 2010 \\
\hline P. fluorescens & M. incognita & Mulberry & Muthulakshmi and Devrajan 2015 \\
\hline P. fluorescens & M. incognita & Tomato & Abo-Elyousr et al. 2010 \\
\hline Pseudomonas sp. & M. incognita & Okra & Vetrivelkalai et al. 2010 \\
\hline
\end{tabular}

many products of fungi or bacteria used as soil conditioners, plant growth promoters, or plant strengtheners are not considered as bio-nematicides even though such outputs may increase plants' ability to tolerate nematode attack (Wilson and Jackson 2013).

It goes without saying that the most successful product should be accepted by growers/end users. In order to achieve satisfaction of such users, more research, especially on the biology, ecology, interaction with other agricultural inputs, and mode of action of these fungal and bacterial biocontrol agents are needed when used as nematicides. Admittedly, such research priorities may call for further development of specialized techniques and realization of growers by merits and demerits of biocontrol agents. The end users should be adequately taught to optimize and adapt to suit their needs for sustainable and environmentally friendly PPN management tactics. Such information is essential for a realistic appraisal of the impact of molecular techniques to enhance their biocontrol potential and monitor their survival and efficacy aiming at developing advanced strategies for PPN control.

\section{Conclusions}

We have evaluated the different strategies of using fungi and bacteria in integrated management of plant-parasitic nematodes. This is a hot issue of present and future research. However, due to the wide versatility of this area, we consolidated uses of bio-nematicides and other pesticides which should be practiced on a wider basis; bio-nematicides can act synergistically or additively with other agricultural inputs in integrated pest management programs. Our presentation as a professional review article and meta-analysis study indicated research priorities for utilizing fungal and bacterial nematicides in sustainable agriculture.

\section{Acknowledgements}

This study was supported in part by the US-Egypt Project cycle 17 (no. 172) and NRC in-house project entitled "Pesticide alternatives against soilborne pathogens attacking legume cultivation in Egypt".

\section{Availability of data and materials}

The data sets supporting the conclusions of this article are included within the article. 


\section{Authors' contributions}

Both authors read and approved the final manuscript. They contributed according to the order of authors.

\section{Ethics approval and consent to participate}

Not applicable

\section{Consent for publication}

Not applicable

\section{Competing interests}

The authors declare that they have no competing interests.

\section{Publisher's Note}

Springer Nature remains neutral with regard to jurisdictional claims in published maps and institutional affiliations.

\section{Author details}

${ }^{1}$ Plant Pathology Department, National Research Centre, El-Behoos St., Dokki, Giza 12622, Egypt. ²Division of Entomology, Sher-e-Kashmir University of Agricultural Sciences and Technology, Shalimar, Srinagar, Jammu and Kashmir, India.

\section{Received: 9 February 2018 Accepted: 7 September 2018} Published online: 24 September 2018

\section{References}

Aalten PM, Vitour D, Blanvillian D, Gowen SR, Sutra L (1998) Effect of rhizosphere fluorescent Pseudomonas strains on plant parasitic nematodes Radopholus similis and Meloidogyne spp. Letters Appl Microbiol 27:357-361

Abd-Elgawad MMM (2014) Plant-parasitic nematode threats to global food security. J Nematol 46:130

Abd-Elgawad MMM (2016) Comments on the use of biocontrol agents against plant-parasitic nematodes. Int J PharmTech Res 9:352-359

Abd-Elgawad MMM, Askary TH (2015) Impact of phytonematodes on agriculture economy. In: Askary TH, Martinelli PRP (eds) Biocontrol Agents of Phytonematodes. CAB International, Wallingford, pp 3-49

Abd-Elgawad MMM, Vagelas IK (2015) Nematophagous bacteria: field application and commercialization. In: Askary TH, Martinelli PRP (eds) Biocontrol Agents of Phytonematodes. CAB International, Wallingford, pp 276-309

Abo-Elyousr KA, Khan Z, El-Morsi Award M, Abdel-Moneim MF (2010) Evaluation of plant extracts and Pseudomonas spp. for control of root-knot nematode, Meloidogyne incognita on tomato. Nematropica 40:89-99

Adiko A, Gowen SR (1994) Comparison of two inoculation methods of root-knot nematodes for the assessment of biocontrol potential of Pasteuria penetrans. Afro-Asian J Nematol 4:32-34

Ahmed R (1990) Studies on the efficacy of Pasteuria penetrans for the biological control of Meloidogyne species, Ph.D. dissertation. University of Reading, Reading

Ahmed R, Abbas MK, Khan MA, Inam ul Haq M, Javed N, Sahi ST (1994) Evaluation of different methods of application of Pasteuria penetrans for the biocontrol of root-knot of tomato (Meloidogyne incognita). Pakistan J Nematol 12:155-160

Akhtar A, Hisamuddin R, Abbasi S (2012) Interaction between Meloidogyne incognita, Pseudomonas fluorescens and Bacillus subtilis and its effect on plant growth of black gram (Vigna mungo L.). Int J PI Path 3:66-73

Al-Hazmi AS, Javeed MT (2016) Effects of different inoculum densities of Trichoderma harzianum and Trichoderma viride against Meloidogyne javanica on tomato. Saudi J Biol Sci 23:288-292

Amin AW (2000) Efficacy of Arthrobotrys oligospora, Hirsutella rhossiliensis, Paecilomyces lilacinus and Pasteuria penetrans as potential biocontrol agents against Meloidogyne incognita on tomato. Pakistan J Nematol 18 : 29-33

Anita B, Samiyappan R (2012) Induction of systemic resistance in rice by Pseudomonas fluorescens against rice root-knot nematode Meloidogyne graminicola. J Biopesticides 5:53-59

Anita E, Rajendran G (2002) Nursery application of Pseudomonas fluorescens for the control of Meloidogyne incognita on tomato and brinjal. Nematol Medit 30:209-210

Anonymous (1995) Annual Report 1994-95. Indian Institute of Spices Research (IISR). Calicut, India, p 89
Anonymous (2018) Poncho/NOTiVO. Available at: https://agriculture.basf.com/us/ en/Crop-Protection/Poncho-VOTiVO.html (Accessed 30 June 2018)

Anver S (2003) Effect of different organic amendments with Paecilomyces lilacinus for the management of soil nematodes. Archiv Phytopath PI Prot 36:103-109

Anver S, Alam MM (1997) Control of Meloidogyne incognita and Rotylenchulus reniformis singly and concomitantly on pigeonpea with Paecilomyces lilacinus. Indian J Nematol 27:209-213

Anver S, Alam MM (1999) Control of Meloidogyne incognita and Rotylenchulus reniformis singly and concomitantly on chickpea and pigeonpea. Archiv Phytopath PI Prot 32:161-172

Ashoub AH, Amara MT (2010) Biocontrol activity of some bacterial genera against root-knot nematode Meliodogyne incognita. J American Sci 6:321-328

Ashraf MS, Khan TA (2008) Biomanagement of reniform nematode, Rotylenchulus reniformis by fruit wastes and Paecilomyces lilacinus on chickpea. World J Agric Sci 4:492-494

Ashraf MS, Khan TA (2010) Integrated approach for the management of Meloidogyne javanica on eggplant using oil cakes and biocontrol agents. Archiv Phytopath PI Prot 43:609-614

Askary TH (1996) Studies on some nematophagous fungi in agriculture soil of Pusa Farm, Samastipur, Bihar. M.Sc. (Ag.) Thesis, Department of Nematology, Rajendra Agricultural University, Pusa, Bihar, India

Askary TH (2008) Studies on root-knot nematode infesting pigeonpea and its integrated management. PhD Thesis, Department of Plant Protection, Faculty of Agricultural Sciences, Aligarh Muslim University, Aligarh, India

Askary TH (2012) Management of root-knot nematode Meloidogyne javanica in pigeonpea through seed treatment. Indian J Ecol 39:151-152

Askary TH (2015a) Nematophagous fungi as biocontrol agents of phytonematodes. In: Askary TH, Martinelli PRP (eds) Biocontrol Agents of Phytonematodes. CAB International, Wallingford, pp 81-125

Askary TH (2015b) Limitations, research needs and future prospects in the biological control of phytonematodes. In: Askary TH, Martinelli PRP (eds) Biocontrol agents of phytonematodes. CAB International, Wallingford, pp 446-454

Askary TH, Martinelli PRP (2015) Biocontrol agents of phytonematodes. CAB International, Wallingford, p 470

Baheti BL, Dodwadiya M, Bhati SS (2017) Eco-friendly management of maize cyst nematode, Heterodera zeae on sweet corn (Zea mays L. saccharata). J Entomol Zool Stud 5:989-993

Baidoo R, Mengistu T, McSorley R, Stamps RH, Brito J, Crow WT (2017) Management of root-knot nematode (Meloidogyne incognita) on Pittosporum tobira under greenhouse, field, and on-farm conditions in Florida. J Nematol 49:133-139

Bhardwaj P, Trivedi PC (1996) Biological control of Heterodera avenae on wheat using different inoculum levels of Verticillium chlamydosporium. Ann PI Prot Sci 4:111-114

Bhat MY, Hisamuddin R, Bhat NA (2009) Histological interactions of Paecilomyces lilacinus and Meloidogyne incognita on bitter gourd. J American Sci 5:8-12

Bhat MY, Wani AH (2012) Bio-activity of fungal culture filtrates against root-knot nematode egg hatch and juvenile motility and their effects on growth of mung bean (Vigna radiata L. Wilczek) infected with the root-knot nematode, Meloidogyne incognita. Archiv Phytopath PI Prot 45:1059-1069

Bhatt J, Chaurasia RK, Sengupta SK (2002a) Management of Meloidogyne incognita by Paecilomyces lilacinus and influence of different inoculums levels of Rotylenchulus reniformis on betelvine. Indian Phytopath 55:348-350

Bhatt J, Sengupta SK, Chaurasia RK (2002b) Management of Meloidogyne incognita by Trichoderma viride in betelvine. Indian Phytopath 55:97-98

Bhattacharya D, Swarup G (1988) Pasteuria penetrans, a pathogen of the genus Heterodera, its effect on nematode biology and control. Indian J Nematol 18:61-70

Bokhari FM (2009) Efficacy of some Trichoderma species in the control of Rotylenchulus reniformis and Meloidogyne javanica. Archiv Phytopath PI Prot 42:361-369

Brahma U, Borah A (2016) Management of Meloidogyne incognita on pea with bioagents and organic amendment. Indian J Nematol 46:58-61

Brown SM, Kepner JL, Smart GC Jr (1985) Increased crop yields following application of Bacillus penetrans to field plots infested with Meloidogyne incognita. Soil Biol Biochem 17:483-486

Brown SM, Nordmeyer D (1985) Synergistic reduction in root galling by Meloidogyne javanica with Pasteuria penetrans and nematicide. Revue de Nemat 8:285-286

Brown SM, Smart GC Jr (1985) Root penetration by Meloidogyne incognita juveniles infected with Bacillus penetrans. J Nematol 17:123-126 
Cabanillas E, Barker KR (1989) Impact of Paecilomyces lilacinus inoculums level of Meloidogyne incognita on tomato. J Nematol 21:115-120

Cabanillas E, Barker KR, Nelson LA (1989) Survival of Paecilomyces lilacinus in selected carries and related effects on Meloidogyne incognita on tomato. J Nematol 21:121-130

Candanedo-Lay E, Lara J, Jatala P, Gonzales F (1982) Preliminary evaluation of Paecilomyces lilacinus as a biological control of root-knot nematode Meloidogyne incognita in industrial tomatoes. Nematropica 12:154

Chand R, Gill JS (2002) Evaluation of various application methods of Pasteuria penetrans against Meloidogyne incognita in tomato. Indian J Nematol 32:23-25

Chatali L, Singh S, Goswami BK (2003) Effect of cakes with Trichoderma viride for the management of disease complex caused by Rhizoctonia bataticola and Meloidogyne incognita on okra. Ann PI Prot Sci 11:178-180

Chaudhary KK, Kaul RK (2013) Efficacy of Pasteuria penetrans and various oil seed cakes in management of Meloidogyne incognita in chili pepper (Capsicum annuum L.). J Agric Sci Tech 15:617-626

Chen ZX, Dickson DW, McSorley R, Mitchell DJ, Hewlett TE (1996) Suppression of Meloidogyne arenaria race 1 by soil application of endospores of Pasteuria penetrans. J Nematol 28:159-168

Chen ZX, Dickson DW, Mitchell DJ, McSorley R, Hewlett TE (1997) Suppression mechanism of Meloidogyne arenaria race 1 by Pasteuria penetrans. J Nematol 29:1-8

Cho MR, Na SY, Yiem MS (2000) Biological control of Meloidogyne arenaria by Pasteuria penetrans. J Asia-Pacific Entom 3:71-76

Ciancio A (1995) Density dependent parasitism of Xiphinema diversicaudatum by Pasteuria penetrans in a naturally infested field. Phytopathology 85:144-149

Ciancio A, Bourijate M (1995) Relationship between Pasteuria penetrans infection levels and density of Meloidogyne javanica. Nemat Medit 23:43-49

Crow WT, Luc JE, Giblin-Davis RM (2011) Evaluation of Econem ${ }^{\mathrm{TM}}$, a formulated Pasteuria sp. bionematicide, for management of Belonolaimus longicuadatus on golf course turf. J Nematol 43:10-109

Dababat AA, Sikora RA (2007) Use of Trichoderma harzianum and Trichoderma viride for the biological control of Meloidogyne incognita on tomato. J Agric Sci 3:297-309

Dababat AA, Sikora RA, Hauschild R (2006) Use of Trichoderma harzianum and Trichoderma viride for biological control of Meloidogyne incognita on tomato. Comm Agric Appl Biol Sci 71:953-961

Daudi AT, Channer AG, Ahmed R, Gowen SR (1990) Pasteuria penetrans as a biocontrol agent of Meloidogyne javanica in the field in Malawi and in microplots in Pakistan. Proc Brighton Crop Prot Conf. Brighton, UK. 1 : 253-257

Daudi AT, Gowen SR (1992) The potential for managing root-knot nematodes by use of pasteuria penetrans and oxamyl. Nematol Medit 20:241-244

Davide RG, Zorilla RA (1986) Evaluation of a fungus Paecilomyces lilacinus for the biological control of Meloidogyne incognita on okra compared with a nematicide Isazofos. Int Nematol Network Newslet 3:32-33

De Channer AG (1989) The potential of Pasteuria penetrans for the biological control of Meloidogyne species, Ph.D. dissertation. University of Reading, Reading

De Leij FAAM, Davies KG, Kerry BR (1992) The use of Verticillium chlamydosporium and Pasteuria penetrans alone and in combination to control Meloidogyne incognita on tomato plants. Fund Appl Nematol 15:235-242

De Leij FAAM, Kerry BR, Dennehy JA (1993) Verticillium chlamydosporium as a biocontrol agents for Meloidogyne incognita and M. hapla in pot and microplot tests. Nematologica 39:115-126

Deacon J (2018) The microbial world. Available at: http://archive.bio.ed.ac.uk/ jdeacon/microbes/catenar.htm (Accessed 30 June 2018)

Deka R, Sinha AK, Neog PP (2002) Effect of Paecilomyces lilacinus and botanicals against Tylenchulus semipenetrans on Citrus jambhiri. Indian J Nematol 32: 230-231

Deori R, Borah A (2016) Efficacy of Glomus fasciculatum, Trichoderma harzianum for the management of Meloidogyne incognita and Rhizoctonia solani disease complex in green gram. Indian J Nematol 46:61-64

Devapriyanga R, Jonathan El, Sankarimeena K, Kavitha PG (2012) Bioefficacy of Pseudomonas and Bacillus isolates against root-knot nematode, Meloidogyne incognita in black pepper cv. Panniyur 1. Indian J Nematol 42:57-65

Devi LS, Dutta U (2002) Effect of Pseudomonas fluorescens on root-knot nematodes, (Meloidogyne incognita) on okra plant. Indian J Nematol 32: 215-216

Devi LS, Hassan MG (2002) Effect of organic manure singly and in combination with Trichoderma viride against root-knot nematode (Meloidogyne incognita) of soybean (Glycin max L. Mrill). Indian J Nematol 32:190-192
Devi LS, Sharma R (2002) Effect of Trichoderma spp. against root-knot nematodes, Meloidogyne incognita on tomato. Indian J Nematol 32:227-228

Devi TS, Mahanta B, Borah A (2016) Comparative efficacy of Glomus fasciculatum, Trichoderma harzianum, carbofuran and carbendazim in management of Meloidogyne incognita and Rhizoctonia solani disease complex on brinjal. Indian J Nematol 46:161-164

Devrajan K, Rajendran G (2002) Effect of fungal egg parasite Paecilomyces lilacinus (Thom.) Samson on Meloidogyne incognita in banana. Indian J Nematol 32:88-90

Dhawan SC, Singh S (2009) Compatibility of Pochonia chlamydosporia with nematicide and neem cake against root-knot nematode, Meloidogyne incognita infesting okra. Indian J Nematol 39:85-89

Dhawan SC, Singh S (2010) Management of root-knot nematode, Meloidogyne incognita using Pochonia chlamydosporia on okra. Indian J Nematol 40:171-178

Dhawan SC, Singh S, Kamra A (2008) Bio-management of root-knot nematode, Meloidogyne incognita by Pochonia chlamydosporia and Pseudomonas fluorescens on brinjal in farmer's field. Indian J Nematol 38:110-111

Dhawan SS, Narayana R, Babu NP (2004) Biomanagement of root-knot nematode, Meloidogyne incognita in okra by Paecilomyces lilacinus. Ann PI Prot Sci 12:356-359

Dube B, Smart GC Jr (1987) Biological control of Meloidogyne incognita by Paecilomyces and Pasteuria penetrans. J Nematol 19:222-227

Duponnois R, Netscher C, Mateille T (1997) Effect of rhizosphere microflora on Pasteuria penetrans parasitizing Meloidogyne graminicola. Nemat Medit 25:99-103

Dwivedi K, Upadhyay KD, Verma RA, Ahmad F (2008) Role of bioagents in management of Pratylenchus thornei infecting chickpea. Indian J Nematol 38: $138-140$

Eapen SJ, Ramana KV, Sarma YR, Edison S, Sasikumar B, Babu KN (1996) Evaluation of Pseudomonas fluorescens isolates for control of Meloidogyne incognita in black pepper (Piper nigrum L.). Proc Biotech spices, medic aromat pl, Calicut, India, 24-25 Apr, pp.129-133

Eapen SJ, Venugopal MN (1995) Field evaluation of Trichoderma spp. and Paecilomuces lilacinus for control of root knot nematodes and fungal diseases of cardamom nurseries. Indian J Nematol 25:15-16

Ebrahim A, Seddighe F, Hassan RE (2008) Potential for biocontrol of Heterodera schachtii by Pochonia chlamydosporia var. chlamydosporia on sugarbeet. Biocont Sci Tech 18:157-167

Eissa MFM, Abd-Elgawad MMM (2015) Nematophagous bacteria as biocontrol agents of phytonematodes. In: Askary TH, Martinelli PRP (eds) Biocontrol Agents of Phytonematodes. CAB International, Wallingford, pp 217-243

Eltayeb FME (2017) Biological control of root knot disease of tomato caused by Meloidogyne javanica using Pseudomonas fluorescens bacteria. Int J Cur Microbiol Appl Sci 6:1176-1182

Esfahani MN, Pour BA (2006) The effects of Paecilomyces lilacinus on the pathogenesis of Meloidogyne javanica and tomato plant growth parameters. Iran Agric. Res 24:67-75

Feyisa B, Lencho A, Selvaraj T, Getaneh G (2015) Evaluation of some botanicals and Trichoderma harzianum for the management of tomato root-knot Nematode (Meloidogyne incognita (Kofoid and White) Chitwood. Adv Crop Sci Tech 4:201. doi:https://doi.org/10.4172/2329-8863.1000201

Feyisa B, Lencho A, Selvaraj T, Getaneh G (2016) Evaluation of some botanicals and Trichoderma harzianum against root-knot nematode Meloidogyne incognita (Kofoid and White) Chitwood in tomato. J Entomol Nematol 8:11-18

Ganaie MA, Khan TA (2010) Biological potential of Paecilomyces lilacinus on pathogenesis of Meloidogyne javanica infecting tomato plant. European $J$ Appl Sci 2:80-84

Glare TR, Caradus J, Gelernter W, Jackson T, Keyhani N, KÖhl J, Marrone P, Morin L, Stewart A (2012) Have biopesticides come of age? Trends in Biotech 30:250-258

Gogoi BB, Gill JS (2001) Compatibility of Pasteuria penertrans with carbofuran and organic amendments, its effect on Heterodera cajani. Ann PI Prot Sci 9:254-257

Gogoi D, Mahanta B (2013) Comparative efficacy of Glomus fasciculatum, Trichoderma harzianum, carbofuran and carbendazim in management of Meloidogyne incognita and Rhizoctonia solani disease complex on French bean. Ann PI Prot Sci 21:172-175

Gopinatha KV, Gowda DN, Nagesh M (2002) Management of root-knot nematode Meloidogyne incognita on tomato using bioagent Verticillium chlamydosporium, neem cake, marigold and carbofuran. Indian J Nematol 32:179-181

Goswami BK, Mittal A (2004) Management of root-knot nematode infecting tomato by Trichoderma viride and Paecilomyces lilacinus. Indian Phytopath 57:235-236 
Goswami BK, Pandey RK, Rathour KS, Bhattacharya C, Singh L (2006) Integrated application of some compatible biocontrol agents along with mustard oil seed cake and furadan on Meloidogyne incognita infecting tomato plants. J Zhejiang Univ Sci 7:873-875

Goswami BK, Singh S (2002) Effect of Aspergillus niger and cladosporium oxysporum on root-knot nematode (Meloidogyne incognita) multiplication on eggplant. Indian J Nematol 32:94-96

Hallmann J, Quadt-Hallmann A, Rodríguez-Kábana R, Kloepper JW (1998) Interactions between Meloidogyne incognita and endophytic bacteria in cotton and cucumber. Soil Biol Biochem 30:925-937

Hanna Al, Riad FW, Tawfik AE (1999) Efficacy of antagonistic rhizobacteria on the control of root-knot nematode, Meloidogyne incognita in tomato plants. Egyptian J. Agric Res 77:1467-1476

Hasan N (2004) Evaluation of native strain of Paecilomyces lilacinus against Meloidogyne incognita in cowpea followed by Lucerne. Ann PI Prot Sci 3:145-148

Haseeb A, Kumar V, Abuzar S, Sharma A (2007) Integrated management of Meloidogyne incognita-Sclerotinia sclerotiorum disease complex of Mentha arvensis cv. Gomti by using Trichoderma species, neem seed powder, carbofuran and topsin-M. 7th National Symposium on plant Protection Options Implementation and Feasibility, 20-22 Dec. pp. 102

Hewlett TE, Dickson DW, Mitchell DJ, Kannwischer-Mitchell ME (1988) Evaluation of Paecilomyces lilacinus as a biocontrol agent of Meloidogyne javanica on tobacco. J Nematol 20:578-584

Hussain S, Zareen A, Zaki MJ, Abid M (2001) Response of ten chickpea (Cicer arietinum L.) cultivars against Meloidogyne javanica (Treub) Chitwood and disease control by fungal filtrates. Pakistan J Biol Sci 4:429-432

Jamshidnejad V, Sahebani N, Etebarian H (2013) Potential biocontrol activity of Arthrobotrys oligospora and Trichoderma harzianum Bl against Meloidogyne javanica on tomato in the greenhouse and laboratory studies. Archiv Phytopath PI Prot 46:1632-1640

Jansson H-B, Tunlid A, Nordbring-Hertz B (1997) Biological control: nematodes. In: Fungal biotechnology.Anke T (ed.). Chapman and Hall, Weinheim, pp 38-50

Jatala P, Kaltenbach R, Bocangel M (1979) Biological control of Meloidogyne incognita acrita and Globodera pallida on potatoes. J Nematol 11:303

Jatala P, Kaltenbach R, Bocangel M, Devaux AJ, Campos R (1980) Field application of Paecilomyces lilacinus for controlling Meloidogyne incognita on potatoes. J Nematol 12:226-227

Javed N, El-Hassan S, Gowen SR, Pemproke B, Inam-ul-Haq M (2008) The potential of combining Pasteuria penetrans and neem (Azadirachta indica) formulations as a management system for root knot nematodes on tomato. European J PI Path 120:53-60

Jayakumar J, Ramakrishnan S, Rajendran G (2002) Effect of culture filtrate of Pseudomonas fluorescens strain PFI on cotton reniform nematode, Rotylenchulus reniformis. Indian J Nematol 32:228-230

Jayakumar J, Ramakrishnan S, Rajendran G (2004) Biological control of cotton reniform nematode, Rotylenchulus reniformis with Pseudomonas fluorescens. Indian J Nematol 34:230-231

Jayaraj MA, Mani A (1988) Biocontrol of Meloidogyne javanica with the bacterial spore parasite Pasteuria penetrans. Int Nematol Network Newslet 5:3-4

Jegathambigai V, Wilson-Wijeratnam RS, Wijesundera RLC (2011) Effect of Trichoderma viride Strain NRRL 6418 and Trichoderma harzianum (Hypocrea lixii TWC1) on Livistona rotundifoliaroot-knot nematodeMeloidogyne incognita. J Entomol 8:229-239

Jonathan El, Barker KR, Abdel-Alim FF, Vrain TC, Dickson DW (2000) Biological control of Meloidogyne incognita on tomato and banana with rhizobacteria actinomycetes, and Pasteuria penetrans. Nematropica 30:231-240

Jonathan El, Cannayane I, Samiyappan R (2004) Field application of biocontrol agents for the management of spiral nematode, Helicotylenchus multicinctus in banana. Nematol Medit 32:169-173

Jonathan El, Padmanabhan D, Ayyamperumal A (1995) Biological control of rootknot nematode on betelvine, Piper betel by Paecilomyces lilacinus. Nematol Medit 23:191-193

Jonathan El, Rajendran G (2000) Biocontrol potential of the fungus Paecilomyces lilacinus against root-knot nematode Meloidogyne incognita in banana. J Biol Cont 14:67-69

Jonathan El, Sandeep A, Cannayane I, Umamaheswari R (2006) Bioefficacy of Pseudomonas fluorescens on Meloidogyne incognita in banana. Nematol Medit 34:19-25

Jothi G, Sivakumar M, Rajendran G (2003) Management of root-knot nematode by Pseudomonas fluorescens in tomato. Indian J Nematol 33:87-88
Kalaiarasan P, Lakhsmanan PL, Samiyappan R (2010) Induction of oxidative enzyme, peroxidise in groundnut (Arachis hypogaea) by application of Pseudomonas fluorescens as a defence against the root-knot nematode, Meloidogyne arenaria. Indian J Nematol 40:55-59

Kasumimoto T, Ikeda R, Kawada H (1993) Dose response of Meloidogyne incognita infected cherry tomatoes to application of Pasteuria penetrans. Japanese J Nematol 23:10-18

Kavitha J, Jonathan El, Umamaheswari R (2007) Field application of Pseudomonas fluorescens, Bacillus subtilis and Trichoderma viride for the control of Meloidogyne incognita (Kofoid and White) Chitwood on sugarbeet. J Biol Cont 21:211-215

Khalil MS, Allam AFG, Barakat AST (2012b) Nematicidal activity of some biopesticide agents and microorganisms against root-knot nematode on tomato plants under greenhouse conditions. J PI Prot Res 52:47-52

Khalil MS, Kenawy A, Gohrab MA, Mohammed EE (2012a) Impact of microbial agents on Meloidogyne incognita management and morphogenesis of tomato. J Biopesticides 5:28-35

Khan A, Williams KL, Nevalainen HKM (2006) Control of plant-parasitic nematodes by Paecilomyce lilacinus and Monacrosporium lysipagum in pot trials. Biocontrol 51:643-658

Khan MR, Goswami BK (2000) Effect of different doses of Paecilomyces lilacinus isolate 6 on Meloidogyne incognita infecting tomato. Indian J Nematol 30:5-7

Khan MW, Esfahani MN (1990) Efficacy of Paecilomyces lilacinus for controlling Meloidogyne javanica on tomato in green house in India. Pakistan J Nematol 8:95-96

Khan TA, Azam MF, Hussain SI (1984) Effect of fungal filtrates of A. niger and Rhizoctonia solani on penetration and development of root-knot nematodes and plant growth of tomato var. Marglobe. Indian J Nematol 14:106-109

Khattak S, Khattak B (2011) Management of root-knot nematode with Trichoderma harzianum and spent mushroom compost. Proceedings $46^{\text {th }}$ Croatian and $6^{\text {th }}$ International Symposium on Agriculture held on $14^{\text {th }}-18^{\text {th }}$ February, 2011 at Opatija, Croatia. pp. 157-160

Kokalis-Burelle N (2015) Pasteuria penetrans for control of Meloidogyne incognita on tomato and cucumber, and $M$. arenaria on snapdragon. J Nematol 47:207-213

Korayem AM, Hasabo SA, Ameen HH (1993) Effects and mode of action of some plant extracts on certain plant parasitic nematodes. Anz Schädling 66:32-36

Krishnaveni M, Subramanian S (2004) Evaluation of biocontrol agents for the management of Meloidogyne incognita on cucumber (Cucumis sativus L). Cur Nematol 15:33-37

Kumar A, Walia RK, Kapoor A (2005b) Field evaluation of Pasteuria penetrans as nursery bed application against Meloidogyne javanica infecting brinjal. Int J Nematol 15:183-186

Kumar D, Bhatt J, Sharma RL (2017) Efficacy of different bio control agents against Meloidogyne incognita and Fusarium oxysporum on black gram (Vigna mungo L). Int J Cur Microbiol Appl Sci 6:2287-2291

Kumar P, Chand R (2015) Bioefficacy of trichoderma harzianum against root-knot nematode Meloidogyne incognita on brinjal. Ann PI Prot Sci 23:361-364

Kumar PS, Jonathan El, Samiyappan R (2008) Field application of biocontrol agent, Pseudomonas fluorescens for the management of burrowing nematode, Radopholus similis in banana. Indian J Nematol 38:57-61

Kumar S, Khanna AS (2006) Role of Trichoderma harzianum and neem cake separately and in combination against root-knot nematode on tomato. Indian J Nematol 36:264-266

Kumar S, Prabhu S (2008) Biological control of Heterodera cajani in pigeonpea by Trichoderma harzianum and Pochonia chlamydosporia. Indian J Nematol 38:65-67

Kumar T, Kang SC, Maheshwari DK (2005a) Nematicidal activity of some fluorescent Pseudomonads on cyst forming nematode, Heterodera cajani and growth of Sesamum indicum var. RT1. Agric Chem Biotech 48:161-166

Kumar V, Jain RK (2010a) Management of root-knot nematode, Meloidogyne incognita using Pochonia chlamydosporium on okra. Indian J Nematol 40:171-178

Kumar V, Jain RK (2010b) Management of root-knot nematode, Meloidogyne incognita, by Trichoderma viride, T. harzianum and bacterial antagonist, Pseudomonas fluorescens as seed treatment on okra. Indian J Nematol 40 226-228

Kumar V, Singh RV, Singh HS (2011) Management of Meloidogyne incognita race 1 and Rotylenchulus reniformis by seed treatment with biological agents, organic cakes and pesticides on cowpea. Ann PI Prot Sci 19:164-167

Li S, Duan YX, Zhu XF, Chen LJ, Wang YY (2011) The effects of adding secondary metabolites of Aspergillus niger on disease resistance to root-knot nematode of tomato. China Vegetables 1:44-49 
Mahanta B, Borbora AC, Konwar B (2016) Effect of Paecilomyces lilacinus on Tylenchulus semipenetrans population in Khasi Mandarin. Indian J Nematol 46:55-81

Maheswari TU, Mani A (1989) Combined efficacy of Pasteuria penetrans and Paecilomyces lilacinus on the biocontrol of Meloidogyne javanica on tomato. Int Nematol Network Newslet 5:10-11

Mani MP, Rajeswari S, Siva Kumar CV (1998) Management of the potato cyst nematodes, Globodera spp. through plant rhizosphere bacterium Pseudomonas fluorescens Migula. J Biol Cont 12:131-134

Mankau R, Prasad N (1972) Possibilities and problems in the use of a sporozoan endoparasite for biological control of plant parasitic nematodes. Nematropica 2:7-8

Manzoor SM, Sinha AK, Bora BC (2002) Management of citrus nematode, Tylenchulus semipenetrans on Khasi Mandarin, by Paecilomyces lilacinus. Indian J Nematol 32:153-155

Mehtab A, Javed N, Khan SA, Gondal AS (2013) Combined effect of Pasteuria penetrans and neem extract on the development of root-knot nematode in medicinal plants. Pakistan J Nematol 31:55-59

Mendoza A, Sikora RA, Kiewnick S (2004) Efficacy of Paecilomyces lilacinus (strain 251) for the control of Radopholus similis in banana. Comm Agric Appl Biol Sci 69:365-372

Meyer SF, Roberts DP, Chitwood DJ, Carta LK, Lumsden RD, Mao W (2001) Application of Burkholderia cepacia and Trichoderma virens, alone and in combinations, against Meloidogyne incognita on bell pepper. Nematropica 31:75-86

Mokbel AA, Obad IM, Ibrahim IKA (2009) The role of antagonistic metabolites in controlling root-knot nematode, Meloidogyne arenaria on tomato. Alexandria J Agric Res 54:199-205

Muthulakshmi M, Devrajan K (2015) Management of Meloidogyne incognita by Pseudomonas fluorescens and Trichoderma viride in mulberry. Int J PI Prot 8:1-6

Muthulakshmi M, Devrajan K, Jonathan El (2010) Biocontrol of root-knot nematode, Meloidogyne incognita (Kofoid and White) Chitwood in mulberry (Morus alba L.). J Biopesticides 3:479-482

Muthulakshmi M, Kumar S, Subramanian S, Anita B (2012) Compatibility of Pochonia chlamydosporia with other biocontrol agents and carbofuran. J Biopesticides 5:243-245

Nagesh M, Hussaini SS, Singh SP, Biswas SR (2003) Management of root-knot nematode, Meloidogyne incognita (Kofoid and White) Chitwood in chrysanthemum using Paecilomyces lilacinus (Thom) Samson in combination with neem cake. J Biol Cont 17:125-131

Nagesh M, Jankiram T (2004) Root-knot nematode problem in polyhouse roses and its management using dazomat, neem cake and Pochonia chlamydosporia (Verticillium chlamydosporium). J Ornament Hort, New Series 7:147-152

Nagesh M, Parvatha Reddy P, Rama N (2001) Influence of oil cakes in combination with inorganic fertilizers on growth and sporulation of Paecilomyces lilacinus and its antagonism on Meloidogyne incognita infecting tomato. Nematol Medit 29:23-27

Nama CP, Sharma HK (2017) Bio-management of root-knot nematode, Meloidogyne incognita on cowpea (Vigna unguiculata L.). J Entomo Zool Stud 5:50-52

Narasimhamurthy HB, Ravindra H, Sehgal M (2017a) Management of rice rootknot nematode, Meloidogyne graminicola. Int J Pure Appl Biosci 5:268-276

Narasimhamurthy HB, Ravindra H, Sehgal M, Ekabote SD, Ganapathi (2017b) Management of rice root-knot nematode, Meloidogyne graminicola. J Entomology and Zoology Studies 5:1433-1439

Naserinasab F, Sahebani N, Etebarian HR (2011) Biological control of Meloidogyne javanica by Trichoderma harzianum Bl and salicylic acid on tomato. African J Food Sci 5:276-280

Nisha MS, Sheela MS (2016) Effect of fungal egg parasite, Paecilomyces lilacinus (Thom.) Samson on Meloidogyne incognita in brinjal. Indian J Nematol 46:157-159

Oclarit EL, Cumagun CJR (2009) Evaluation of efficacy of Paecilomyces lilacinus as biological control agent of Meloidogyne incognita attacking tomato. J PI Prot Res 49:337-340

Oduor-owino P (2003) Integrated management of root-knot nematodes using agrochemicals, organic matter and the antagonistic fungus, Paecilomyces lilacinus in natural field soil. Nematol Medit 31:121-123

Oostendorp M, Dickson DW, Mitchell DJ (1991) Population development of Pasteuria penetrans on Meloidogyne arenaria. J Nematol 23:58-64

Opperman CH, Chang S (1990) Plant-parasitic nematodes acetylcholinesterase inhibition by carbamate and organophosphate nematicides. J Nematol 22: 481-488
Page SLJ, Bridge J (1985) Observations on Pasteuria penetrans as a parasite of Meloidogyne acronea. Nematologica 31:238-240

Pandey G, Pandey RK, Pant H (2003) Efficacy of different levels of Trichoderma viride against rootknot nematode in chickpea (Cicer arietinum L.). Ann PI Protect Sci 11: 101-103

Parihar K, Rehman B, Ganai MA, Asif M, Siddiqui MA (2015) Role of oil cakes and Pochonia chlamydosporia for the management of Meloidogyne javanica attacking Solanum melongena L. J PI Path and Microbiol (Special Issue) SI:1-5. https://doi.org/10.4172/2157-7471.SI-004

Parvatha Reddy P, Khan RM (1988) Evaluation of Paecilomyces lilacinus for the biological control of Rotylenchulus reniformis infecting tomato, compared with cabofuran. Nematol Medit 16:113-115

Parvatha Reddy P, Nagesh M, Devappa V (1997) Effect of integration of Pasteuria penetrans, Paecilomyces lilacinus and neem cake for the management of root-knot nematodes infecting tomato. Pest Manag Hortl Ecosyst 3:100-104

Parvatha Reddy P, Rao MS, Nagesh M (1996) Management of the citrus nematode, Tylenchulus semipenetrans, by integration of Trichoderma harzianum with oil cakes. Nematologia Mediterranea 24:265-267

Pathak B, Khan MR (2010) Comparative field efficacy of chemical, botanical and biological agents against foliar nematode, Aphelenchoides besseyi infecting tuberose. Indian J Nematol 40:83-87

Pathak KN, Kumar B (2003) Effect of culture filtrates of Gliocladium virens and Trichoderma harzianum on the penetration of rice roots by Meloidogyne graminicola. Indian J Nematol 33:149-151

Pathak KN, Ranjan R, Kumar M, Kumar B (2005) Biomanagement of Meloidogyne graminicola by Trichoderma harzianum and T. virens in rice. Ann PI Prot Sci 13:438-440

Perveen S, Ehteshamul-Haque S, Ghaffar A (1998) Efficacy of Pseudomonas aeruginosa and Paecilomyces lilacinus in the control of root rot-root knot disease complex on some vegetables. Nematol Medit 26:209-212

Prakob W, Nguen-Hom J, Jaimasit P, Silapapongpri S, Thanunchai J, Chaisuk P (2009) Biological control of lettuce root knot disease by use of Pseudomonas aeruginosa, Bacillus subtilis and Paecilomyces lilacinus. J Agric Tech 5:179-191

Prasad D, Anes KM (2008) Effect of metabolites of Trichoderma harzianum and T. viride on plant growth and Meloidogyne incognita on okra. Ann PI Prot Sci 16:461-465

Priya MS (2015) Biomanagement of rice root knot nematode, Meloidogyne graminicola Golden and Brichfield in aerobic rice. Int J Manag Soc Sci 3: 591-598

Ramakrishnan S, Nagesh M (2011) Evaluation of beneficial fungi in combination with organics against root-knot nematode, Meloidogyne incognita, in FCV tobacco nurseries. J Biol Cont 25:311-315

Ramakrishnan S, Rao CP (2013) Evaluation of Paecilomyces lilacinus for the management of root-knot nematode, Meloidogyne incognita in flue cured Virginia (FCV) tobacco nursery. Indian J Nematol 43:65-69

Ramakrishnan S, Sivakumar CV, Poornima K (1998) Management of rice root nematode, Hirschmanniella gracilis (de Man) Luc and Goodey with Pseudomonas fluorescens Migula. J Biol Cont 12:135-141

Rangaswamy SD, Parvatha Reddy P, Nagesh M (2000) Evaluation of biocontrol agents ( $P$. penetrans and $T$. viride) and botanicals for the management of root-knot nematode, M. incognita infecting tomato. Pest Manag Hort Ecosyst 6:135-138

Rao MS (2007) Papaya seedlings colonized by the bio-agents Trichoderma harzianum and Pseudomonas fluorescens to control root-knot nematodes. Nematol Medit 35:199-203

Rao MS, Dhananjay N, Shylaja M (2003) Management of Meloidogyne incognita on eggplant using a formulation of Pochonia chlamydosporia, Zare et al., (Verticillium chlamydosporium). Pest Manag Hort Ecosyst 9:71-76

Rao MS, Dhananjay N, Shylaja M (2004) Biointensive management of root-knot nematode on bell pepper using Pochonia chlamydosporia and Pseudomonas fluorescens. Nematol Medit 32:159-163

Rao MS, Parvatha Reddy P, Nagesh M (1997a) Management of root-knot nematode, Meloidogyne incognita on tomato by integration of Trichoderma harzianum with neem cake. Zeitschrift für Pflanzenkrankheiten und Pflanzenschutz 104:423-425

Rao MS, Parvatha Reddy P, Nagesh M (1997b) Integration of Paecilomyces lilacinus with neem leaf suspension for the management of root-knot nematodes on eggplant. Nematol Medit 25:249-252

Rao MS, Parvatha Reddy P, Nagesh M (1998) Evaluation of plant based formulations of Trichoderma harzianum for the management of Meloidogyne incognita on eggplant. Nematol Medit 26:59-62 
Raveendra HR, Krishna Murthy R, Mahesh Kumar R (2011) Management of root knot nematode Meloidogyne incognita by using oil cake, bio-agent, trap crop, chemicals and their combination. Int J Sci Nat 2:519-523

Ravichandra NG, Reddy BMR (2008) Efficacy of Pasteuria penetrans in the management of Meloidogyne incognita infecting tomato. Indian J Nematol 38:172-175

Roman J, Rodriguez-Marcano A (1985) Effect of the fungus Paecilomyces lilacinus on the larval population and root-knot formation of Meloidogyne incognita in tomato. J Agric University Puerto Rico 69:159-166

Saikia MK, Roy AK (1994) Efficacy of Paecilomyces lilacinus on the reduction of attack of Meloidogyne incognita on okra. Indian J Nematol 24:163-167

Sandeep A (2004) Bioefficacy of Pseudomonas fluorescens (Native Isolates) on Meloidogyne incognita (Kofoid and White, 1919) Chitwood, 1949 in Banana (Musa spp.). M.Sc. (Ag.) Thesis, Tamil Nadu Agricultural University, Coimbatore, Tamil Nadu, India

Sankaranarayanan C, Hussaini SS, Kumar PS, Rangeshwaran R (2000) Biological control of Meloidogyne incognita (Kofoid and White 1919) Chitwood, 1949 on tomato by Verticillium chlamydosporium Goddard culture on different substrates. J Biol Cont 14:39-43

Santhi A, Sivakumar CV (1995) Biocontrol potential of Pseudomonas fluorescens (Migula) against root-knot nematode, Meloidogyne incognita (Kofoid and White, 1919) Chitwood, 1949 on tomato. J Biol Cont 9:113-115

Santhi A, Sundarababu R, Sivakumar CV, Sundarababu R (1999) Field evaluation of rhizobacterium, Pseudomonas fluorescens for the management of the citrus nematode, Tylenchulus semipenetrans. Procnational symposium on rational approaches in nematode management for sustainable agriculture. Anand, India, 23-25 November, pp. 38-42.

Santin RCM (2008) Potential usage of the fungi Trichoderma spp. and Paecilomyces lilacinus in the control of Meloidogyne incognita and Phaseolus vulgaris. Ph.D. thesis, Universidade Federal do Rio Grande do Sul, Porto Alegre, Brazil.

Seenivasn N, Lakshmanan PL (2002) Biocontrol potential of native isolates of Pseudomonas fluorescens against rice root nematode, Hirschmanniella gracilis. J Ecobiol 15(2):69-72.

Seenivasan N (2007). Integrated management of root-knot nematode Meloidogyne incognita in medicinal Coleus. $7^{\text {th }}$ Nat Sym plant Prot Options Implement Feasibility 20-22 Dec, pp. 100

Seenivasan N, David PMM, Vivekanandan P, Samiyappan R (2012) Biological control of rice root-knot nematode, Meloidogyne graminicola through mixture of Pseudomonas fluorescens strains. Biocont Sci Tech 22:611-632

Seenivasan N, Poornima K (2010) Bio-management of root-knot nematode, Meloidogyne incognita (Kofoid and White) Chitwood in Jasmine (Jasminum sambac L.). Pest Manag Hort Ecosyst 16:34-40

Sekhar NS, Gill JS (1991) Efficacy of Pasteuria penetrans alone and in combination with carbofuran in controlling Meloidogyne incognita. Indian J Nematol 21:61-65

Senthilkumar P, Jonathan El, Samiyappan R (2008) Bioefficacy of Pseudomonas fluorescens on burrowing nematode, Radopholus similis in banana. Indian J Nematol 38:46-52

Shamalie BVT, Fonseka RM, Rajapaksha RGAS (2011) Effect of Trichoderma viride and carbofuran (Curator ${ }^{\oplus}$ ) on management of root-knot nematodes and growth parameters of gotukola (Centella asiatica L.). Trop Agric Res 23:61-69

Shanthi A, Rajeswari S, Sivakumar CV, Mehta UK (1998) Soil application of Pseudomonas fluorescens (Migula) for the control of root knot nematode (Meloidogyne incognita) on grapevine (Vitis vinifera Linn.). Nematology: challenges and opportunities in 21st Century. Procthe Third International Symposium of Afro-Asian Society of Nematologists (TISAASN). Sugarcane Breeding Institute (ICAR), Coimbatore, India, 16-19, Apr, 1998. pp. 203-206.

Sharf R, Shiekh H, Syed A, Akhtar A, Robab MI (2014a) Interaction between Meloidogyne incognita and Pochonia chlamydosporia and their effects on the growth of Phaseolus vulgaris. Archiv Phytopath PI Prot 47:622-630

Sharf R, Abbasi A, Akhtar A (2014b) Combined effect of biofertilizers and fertilizer in the management of Meloidogyne incognita and also on the growth of red kidney bean (Phaseolus vulgaris). Int J Plant Pathology 5:1-11

Sharma HK, Kamra A, Pankaj Lal J, Kumar J (2008) Effect of seed treatment with Pseudomonas fluorescens alone and in combination with soil application of carbofuran and neem seed powder against Meloidogyne incognita in okra. Pesticide Res J 20:79-82

Sharma HK, Prasad D, Sharma P (2005) Compatibility of fungal bioagents as seed dressers with carbofuran in okra against Meloidogyne incognita. National Symposium on Recent Advances and Research Priorities in Indian Nematology, pp. 9-11.
Sharma RD (1992) Biocontrol efficacy of Pasteuria penetrans against Meloidogyne javanica. Ciencia Biologica Ecologica e Systematica 12:43-47

Sharon E, Bar EM, Chet I, Herrera EA, Kleifeld O, Spiegel Y (2001) Biological control of the root-knot nematode M. javanica by T. harzianum. Phytopathology 91:687-693

Siddiqui IA, Ehteshamul-Haque S (2000) Effect of Verticillium chlamydosporium and Pseudomonas aeruginosa in the control of Meloidogyne javanica on tomato. Nematol Medit 28:193-196

Siddiqui IA, Shaukat SS (2003) Suppression of root-knot disease by Pseudomonas fluorescens CHAO in tomato: importance of bacterial secondary metabolite 2,4-diacetylphloroglucinol. Soil Biol Biochem 35:1615-1623

Siddiqui IA, Zareen A, Zaki MJ, Shaukat SS (2001a) Use of Trichoderma species in the control of Meloidogyne javanica, root knot nematode in okra and mungbean. Pakistan J Biol Sci 4:846-848

Siddiqui ZA, labal A, Mahmood I (2001b) Effects of Pseudomonas fluorescens and fertilizers on the reproduction of Meloidogyne incognita and growth of tomato. Appl Soil Ecol 16:179-185

Siddiqui ZA, Mahmood I (1995) Some observations on the management of the wilt disease complex of pigeonpea by treatment with a vesicular arbuscular fungus and biocontrol agents for nematodes. Bioresource Tech 54:227-230

Siddiqui ZA, Mahmood I (1996) Biological control of Heterodera cajani and Fusarium udum on pigeonpea by Glomus mosseae, Trichoderma harzianum, and Verticillium chlamydosporium. Israel J PI Sci 44:49-56

Siddiqui ZA, Mahmood I, Hayat S (1998) Biocontrol of Heterodera cajani and Fusarium udum on pigeonpea using Glomus mosseae, Paecilomyces lilacinus and Pseudomonas fluorescens. Thai J Agric Sci 31:310-321

Siddiqui ZA, Qureshi A, Akhtar MS (2009) Biocontrol of root-knot nematode Meloidogyne incognita by Pseudomonas and Bacillus isolates on Pisum sativum. Archiv Phytopath PI Prot 42:1154-1164

Silva JO, Santana MV, Freire LL, Ferreira BS, Rocha MR (2017) Biocontrol agents in the management of Meloidogyne incognita in tomato. Available at: https:// doi.org/10.1590/0103-8478cr20161053 (Accessed 9 Dec 2017).

Simon LS, Pandey A (2010) Antagonistic efficacy of Paecilomyces lilacinus and Verticillium chlamydosporium against Meloidogyne incognita infecting okra. Indian J Nematol 40:113

Singh B, Dhawan SC (1994) Effect of Pasteuria penetrans on the penetration and multiplication of Heterodera cajani in Vigna unguiculata roots. Nematol Medit 22:159-161

Singh B, Dhawan SC (1996) Suppression of Heterodera cajani by Pasteuria penetrans during three successive plantings of cowpea. Indian J Nematol 26:216-221

Singh B, Dhawan SC (1999) Comparison of different methods of application of Pasteuria penetrans for the control of Heterodera cajani in cowpea. Indian J Nematol 29:118-120

Singh LM, Mahanta B (2013) Effect of carbosulfan, Glomus fasciculatum, Trichoderma harzianum and vermicompost alone and combination in management of Meloidogyne incognita on green gram. Ann PI Prot Sci 21:154-156

Singh M, Singh J, Gill JS (2008) Impact of Pasteuria penetrans on root-knot nematode (Meloidogyne incognita) infecting tomato (Lycopersicon esculentum). Indian J Agric Sci 78:1092-1094

Singh P, Siddiqui ZA (2010) Biocontrol of root-knot nematode Meloidogyne incognita by the isolates of Pseudomonas on tomato. Archiv Phytopath PI Prot 43:1423-1434

Singh S (2013) Integrated approach for the management of the root-knot nematode, Meloidogyne incognita, on eggplant under field conditions. Nematology 15:747-757

Singh SM, Azam MF, Khan AM, Saxena SK (1991) Effect of Aspergillus niger and Rhizoctonia solani on development of Meloidogyne incognita on tomato. Cur Nematol 2:163-166

Sivakumar CV, Bhaskaramanian MD, Balasaraswathy S (1993) Control of root-knot nematode, Meloidogyne araneria in brinjal nursery with fungus, Paecilomyces lilacinus and systemic nematicide carbofuran. J Biol Cont 7:49-50

Somasekhar N, Gill JS (1991) Efficacy of Pasteuria penetrans alone and in combination with carbofuran controlling Meloidogyne incognita. Indian J Nematol 21:61-65

Sosamma VN, Geetha SM, Koshy PK (1994) Effect of the fungus, Paecilomyces lilacinus on the burrowing nematode Radopholus similis infesting betel vine. Indian J Nematol 24:50-53

Spaull WW (1984) Observations on Bacillus penetrans infecting Meloidogyne in sugarcane fields in South Africa. Revue de Nematol 7:277-282

Stevens G, Lewis E (2017) Status of entomopathogenic nematodes in integrated pest management strategies in the USA. In: Abd-Elgawad MMM, Askary TH, 
Coupland J (eds) Biocontrol agents: entomopathogenic and slug parasitic nematodes. CAB International, Wallingford, pp 289-311

Stirling GR (1984) Biological control of Meloidogyne javanica with Bacillus penetrans. Phytopathology 74:55-60

Sudha S, Sundararaju P, lyer R (2000) Effect of Paecilomyces lilacinus for the control of burrowing nematode, Radopholus similis on arecanut seedlings. Indian J Nematol 30:101-103

Thakur NSA, Devi G (2007) Management of Meloidogyne incognita attacking okra by nematophagous fungi, Arthrobotrys oligospora and Paecilomyces lilacinus. Agric Sci Digest 27:50-52

Thakur S, Walia RK (2016) Potential of bacterial parasite, Pasteuria penetrans application as nursery soil treatment and seed treatment in controlling Meloidogyne graminicola infecting rice. Indian J Nematol 46:16-19

Trifonova Z, Tsvetkov I, Bogatzevska N, Batchvarova R (2014) Efficiency of Pseudomonas spp. for biocontrol of the potato cyst nematode Globodera rostochiensis (Woll.). Bulgarian J Agric Sci 20:666-669

Tzortzakakis EA (2007) The effect of the fungus Pochonia chlamydosporia on the root-knot nematode Meloidogyne incognita in pots. Russian J Nematol 15:89-94

Umamaheswari R, Sivakumar M, Subramanian S, Samiyappan R (2004) Induction of systemic resistance by Trichoderma viride treatment in green gram (Vigna radiata) against root-knot nematode Meloidogyne incognita. Cur Nematol 15:1-7

Umamaheswari T, Mani A, Rao PK (1987) Combined efficacy of the bacteria spore parasite, Pasteuria penetrans and nematicides in the control of Meloidogyne javanica on tomato. J Biol Cont 1:53-57

Umamaheswari T, Mani A, Rao PK (1988) Efficacy of the bacterial spore parasite, Pasteuria penetrans and oil Cakes in the control of Meloidogyne javanica on tomato. J Biol Cont 2:34-36

Van Damme V, Hoedekie A, Viaene N (2005) Long-term efficacy of Pochonia chlamydosporia for the management of Meloidogyne javanica in glasshouse crops. Nematology 7:727-736

Vargas R, Acosta N, Moullor A, Betancourt C (1992) Control of Meloidogyne spp. with Pasteuria penetrans (Thorne) Sayre and Starr. J Agric University Puerto Rico 76:63-70

Verdejo-Lucas S (1992) Seasonal population fluctuations of Meloidogyne spp. and the Pasteuria penetrans group in Kiwi orchards. PI Disease 76:1275-1279

Verdejo-Lucas S, Sorribas FJ, Ornat C, Galeano M (2003) Evaluating Pochonia chlamydosporia in a double-cropping system of lettuce and tomato in plastic houses infested with Meloidogyne javanica. PI Path 52:521-528

Verma KK (2009) Management of Meloidogyne javanica by bacterial antagonist, Pseudomonas fluorescens as seedling root dip in tomato. Indian J Nematol 39: 207-210

Vetrivelkalai P, Sivakumar M, Jonathan El (2010) Bio-control potential of endophytic bacteria on Meloidogyne incognita and its effect on plant growth in bhendi. J Biopesticides 3:52-457

Viaene NM, Abawi GS (2000) Hirsutella rhossiliensis and Verticillium chlamydosporium as biocontrol agents of the root-knot nematode Meloidogyne hapla on lettuce. J Nematol 32:85-100

Viggiano JR, Freitas LG, Lopes EA (2015) Pochonia chlamydosporia var. chlamydosporia (Goddard) Zare \& W. Gams for the management of lettuce infected with Meloidogyne javanica (Treub, 1885). Chilean J Agric Res 75:255-258

Vikram, Walia RK (2014) Efficacy of bacterial parasite, Pasteuria penetrans application as nursery soil treatment against root-knot nematode, Meloidogyne javanica infecting tomato in different seasons. Indian J Nematol 44:44-49

Vikram, Walia RK (2015) Efficacy of bacterial parasite, Pasteuria penetrans application as seed treatment against root-knot nematode, Meloidogyne javanica. Indian J Nematol 45:1-6

Wahla V, Maheshwari DK, Bajpai VK (2012) Nematicidal fluorescent pseudomonads for the in vitro and in vivo suppression of root knot (Meloidogyne incognita) of Capsicum annuum L. Pest Manag Sci 68:1148-1155

Walia RK (1994) Assessment of nursery treatment with Pasteuria penetrans for the control of Meloidogyne javanica on tomato in green-house. J Biol Cont 8:68-70

Walia RK, Dalal MR (1994) Efficacy of bacterial parasite, Pasteuria penetrans application as nursery soil treatment in controlling root-knot nematode, Meloidogyne javanica on tomato. Pest Manag Econ Zool 2:19-21

Walia RK, Nandal SN, Bhatti DS (1999) Nematicidal efficacy of plant leaves and Paecilomyces lilacinus alone or in combination in controlling Meloidogyne incognita on okra and tomato. Nematol Medit 27:3-8
Walker GE, Wachtel MF (1989) The influence of soil solarisation and non-fumigant nematicides on infection of Meloidogyne javanica by Pasteuria penetrans. Nematologica 34:477-483

Walters SA, Barker KR (1994) Efficacy of Paecilomyces lilacinus in suppressing Rotylenchulus reniformis on tomato. J Nematol 26:600-605

Wang K, Riggs RD, Crippen D (2005) Isolation, selection, and efficacy of Pochonia chlamydosporia for control of Rotylenchulus reniformis on cotton. Phytopathology 95:890-893

Weibelzahl-Fulton E, Dickson DW, Whitty EB (1996) Suppression of Meloidogyne incognito and Meloidogyne javanica by Pasteuria penetrans in field soil. J Nematol 28:43-49

Wilson MJ, Jackson TA (2013) Progress in the commercialisation of bionematicides. BioCont 58:715-722

Windham GL, Windham MT, Williams WP (1989) Effects of Trichoderma spp. on maize growth and Meloidogyne arenaria reproduction. PI Disease 73:493-495

Zaki MJ, Maqbool MA (1990) Effect of Pasteuria penetrans and Paecilomyces lilacinus on the control of root-knot nematodes of brinjal and mung. Pakistan J Phytopath 2:37-42

Zareen A, Zaki MJ, Ghaffar A (1999) Effect of culture filtrate of fungi in the control of Meloidogyne javanica root-knot nematodes on okra and broad bean. Pakistan J Biol Sci 2:1441-1444

Zareen A, Zaki MJ, Khan NJ (2001) Effect of fungal filtrates of Aspergillus species on development of root-knot nematodes and growth of tomato (Lycopersicon esculentum Mill). Pakistan J Biol Sci 4:995-999

\section{Submit your manuscript to a SpringerOpen ${ }^{\circ}$ journal and benefit from:}

- Convenient online submission

- Rigorous peer review

- Open access: articles freely available online

High visibility within the field

- Retaining the copyright to your article

Submit your next manuscript at $\boldsymbol{\triangleright}$ springeropen.com 